\title{
CLUES TO NUCLEAR STAR CLUSTER FORMATION FROM EDGE-ON SPIRALS
}

\author{
Anil C. Seth, ${ }^{1}$ Julianne J. Dalcanton, ${ }^{2}$ Paul W. Hodge, and Victor P. Debattista ${ }^{3}$ \\ Astronomy Department, University of Washington, Box 351580, Seattle, WA 98195-1580 \\ Received 2006 April 10; accepted 2006 July 3
}

\begin{abstract}
We find nine nuclear cluster candidates in a sample of 14 edge-on, late-type galaxies observed with the Hubble Space Telescope Advanced Camera for Surveys. These clusters have magnitudes $\left(M_{I} \sim-11\right)$ and sizes $\left(r_{\text {eff }} \sim 3 \mathrm{pc}\right)$ similar to those found in previous studies of face-on, late-type spirals and $\mathrm{dE}$ galaxies. However, three of the nuclear clusters are significantly flattened and show evidence for multiple, coincident structural components. The elongations of these three clusters are aligned to within $\sim 10^{\circ}$ of the galaxies' major axes. Structurally, the flattened clusters are well fit by a combination of a spheroid and a disk or ring, with the disk preferred in two of three cases. The nuclear cluster disks/rings have F606W - F814W $(\sim V-I)$ colors 0.3-0.6 mag bluer than the spheroid components, suggesting that the stars in these components have ages $<1$ Gyr. In NGC 4244, the nearest of the nuclear clusters, we further constrain the stellar populations via spectroscopy and multiband photometry. This nuclear cluster is equally well fit by single stellar populations with ages of either $\sim 70 \mathrm{Myr}$ or $\sim 0.8 \mathrm{Gyr}$ and with masses of $\sim 3 \times 10^{6} M_{\odot}$. However, significantly better fits to the spectroscopy and photometry are obtained by combining two or more stellar populations. Exploiting emission lines that appear to originate $\sim 1^{\prime \prime}$ from the NGC 4244 nucleus, we determine a lower limit on the dynamical mass of $2.5_{-1.2}^{+1.7} \times 10^{6} M_{\odot}$ within $19 \mathrm{pc}$, typical of values found for other nuclear clusters. We also present tentative evidence that another of the nuclear clusters (in NGC 4206) may also host a supermassive black hole. Based on our observational results we propose an in situ formation mechanism for nuclear clusters in which stars form episodically in compact nuclear disks and then lose angular momentum or heat vertically to form an older spheroidal structure. We estimate the period between star formation episodes to be $\sim 0.5 \mathrm{Gyr}$ and discuss possible mechanisms for transforming the disklike components into spheroids. We also note the connection between our objects and massive globular clusters (e.g., $\omega$ Cen), ultracompact dwarfs, and supermassive black holes.
\end{abstract}

Key words: galaxies: formation — galaxies: individual (IC 5052, NGC 4244, NGC 4206) — galaxies: nuclei — galaxies: spiral - galaxies: star clusters

Online material: color figures

\section{INTRODUCTION}

Due to their deep gravitational potential wells and unique dynamics, the centers of galaxies frequently host exotic objects such as supermassive black holes (SMBHs), active galactic nuclei (AGNs), and massive stellar clusters. Of these, only nuclear star clusters provide a visible record of the accretion of stars and gas into the nucleus. Therefore, studies of nuclear star clusters hold the promise of shedding light on the formation of central massive objects of all types.

Bright nuclear star clusters have been observed in $\sim 75 \%$ of the 77 face-on galaxies of type Scd and later studied by Böker et al. (2002). Similar clusters appear to be frequent in earlier type spirals as well, although their study is complicated by the presence of bulges and other nuclear phenomena (Carollo et al. 2002). Dwarf elliptical galaxies, which may originate from low-mass spiral galaxies (e.g., Mastropietro et al. 2005; Beasley et al. 2006), also frequently possess nuclear star clusters (Grant et al. 2005; Côté et al. 2006).

In late-type spiral galaxies with little or no bulge, nuclear star clusters are very prominent and can be studied in detail. From their sample of face-on, late-type spiral galaxies, Böker et al. (2004) found that nuclear clusters have dimensions similar to Milky Way globular clusters, with typical half-light radii of $3.5 \mathrm{pc}$. However,

\footnotetext{
${ }^{1}$ Currently a CfA Postdoctoral Fellow, Center for Astrophysics, 60 Garden Street, Cambridge, MA 02138; aseth@cfa.harvard.edu.

${ }^{2}$ Alfred P. Sloan Research Fellow.

${ }^{3}$ Brooks Prize Fellow.
}

these clusters are significantly brighter than globular clusters, with $I$-band magnitudes ranging between -8 and -16 (Böker et al. 2002). Their high luminosities are at least in part due to their large masses; Walcher et al. (2005) have used high-resolution spectra to derive typical masses of $\sim 3 \times 10^{6} M_{\odot}$ for nine galaxies in the Böker et al. (2002) sample, similar in mass to the most massive Milky Way cluster, $\omega$ Cen (Meylan \& Mayor 1986). However, the brightness of nuclear clusters also results in part from the presence of young stellar populations. Photometry and spectra of a number of clusters suggest that nuclear clusters in both spiral and dwarf elliptical galaxies have populations much younger than globular clusters (e.g., Ho et al. 1995; Lotz et al. 2004). In late-type galaxies, most clusters appear to have stars with ages $<100 \mathrm{Myr}$ (Walcher et al. 2006). Furthermore, recent spectral studies have shown that nuclear clusters are made up of composite stellar populations, with most having substantial old ( $\gtrsim 1 \mathrm{Gyr}$ ) stellar components (Long et al. 2002; Sarzi et al. 2005; Walcher et al. 2006; Rossa et al. 2006).

Not only are nuclear clusters present in a large fraction of present-day spiral and dwarf elliptical galaxies, they also have been invoked as the progenitors of massive globular clusters and ultracompact dwarfs (UCDs). In a hierarchical merging scenario, many galaxies will be tidally stripped during mergers, leaving behind only their dense nuclei (e.g., Bekki \& Chiba 2004). Nuclear cluster properties match the half-light radii and exceptional luminosity of massive globular clusters and UCDs (Mackey \& van den Bergh 2005; Phillipps et al. 2001; Jones et al. 2006). Furthermore, the multiple stellar populations seen in $\omega$ Cen and G1 
(e.g., Bedin et al. 2004; Meylan et al. 2001) and the evidence for possible self-enrichment among the brightest globular clusters in elliptical galaxies (Harris et al. 2006) strengthen the association between these bright compact objects.

Finally, nuclear clusters appear to be intimately connected to the formation of SMBHs at the centers of galaxies. For both spiral and elliptical galaxies, a clear correlation is seen between nuclear cluster mass and bulge mass (Côté et al. 2006; Wehner \& Harris 2006; Ferrarese et al. 2006; Rossa et al. 2006). This correlation appears to fall along the well-known $M_{\bullet}-\sigma$ relation between black hole mass and bulge or galaxy mass (e.g., Ferrarese $\&$ Merritt 2000; Ferrarese 2002). Both SMBHs and nuclear clusters appear to contain the same fraction of the total galaxy mass, supporting the idea that they are two related types of central massive object (Wehner \& Harris 2006). Whether a central massive object is a nuclear cluster or a SMBH appears to depend on mass; early-type galaxies more massive than $\sim 10^{10} M_{\odot}$ appear to have SMBHs, while lower mass systems commonly host nuclear clusters (Ferrarese et al. 2006). These findings strongly suggest a similar formation mechanism for nuclear clusters and SMBHs. Increasing evidence also points to massive star clusters as sites of intermediate-mass black hole (IMBH) formation (e.g., Miller \& Colbert 2004; Gebhardt et al. 2005; Patruno et al. 2006). These IMBHs may also follow the $M_{\bullet}-\sigma$ relation (Gebhardt et al. 2002).

In this context, a detailed understanding of how nuclear clusters formed may reveal the general mechanism driving the formation of central massive objects of all types. Two basic scenarios have been suggested (e.g., Böker et al. 2004): (1) nuclear clusters form from multiple globular clusters accreted via dynamical friction (e.g., Tremaine et al. 1975; Lotz et al. 2001), and (2) nuclear clusters form in situ from gas channeled into the center of galaxies (Milosavljević 2004). In this paper we present observational evidence that favors the in situ formation scenario. In particular, we find nuclear clusters that are elongated along the major axis of the galaxies and have two coincident components. These clusters appear to consist of a younger flattened disk or ring and an older spheroid. Based on the evidence we present, we suggest a detailed formation mechanism in which nuclear clusters form in repeated star formation events from very compact nuclear gas disks.

In $\S 2$ we describe the imaging and spectroscopic observations used in the paper. Section 3 focuses on an analysis of the morphology and luminosities of the clusters as revealed by Hubble Space Telescope (HST) observations. The stellar populations of the multiple-component clusters are examined in $\S 4$. In $\S 5$ we derive a dynamical mass for the NGC 4244 nuclear cluster, and then in $\S 6$ we present tentative evidence that the NGC $4206 \mathrm{nu}-$ cleus may harbor both a star cluster and a SMBH. We present a detailed formation mechanism and discuss it in the broader context in $\S 7$, and then we summarize our findings in $\S 8$.

\section{OBSERVATIONS}

\subsection{Identification of Nuclear Clusters}

Most of the data presented in this paper were taken with the HST's Advanced Camera for Surveys (ACS) as part of a Cycle 13 snapshot program. Sixteen galaxies were observed in both the F606W and F814W filters, with exposure times of $\sim 700$ s. Details of these observations and their reduction are presented in Seth et al. (2005, hereafter Paper I).

We identified nuclear clusters by careful examination of the central regions of our galaxies. The exact locations of the galaxy centers were determined using fits to Two Micron All Sky Survey (2MASS) data presented in Paper I. Nuclear clusters were distinguished based on their proximity to the photocenter of the galaxy, their brightness/prominence, and their extent (i.e., if they appeared resolved). At the distance of most of our galaxies, nuclear clusters such as those observed by Böker et al. (2002) should be at least partially resolved.

Table 1 lists the galaxies in our sample and whether each possesses a nuclear cluster (shown in col. [5]). In 2 of the 16 galaxies in the sample, the central region was not covered by the ACS images (denoted by "N/A" in Table 1). Of the remaining 14 galaxies, 7 have prominent and/or extended sources located within $\sim 2^{\prime \prime}$ of the photometric center of the galaxy. Another two have possible detections of nuclear clusters, where the candidate clusters were not very prominent (denoted by "Maybe"). The remaining five galaxies have no obvious candidate nuclear clusters. Of these five definite nondetections, three (IRAS 06070-6147, IRAS 07568-4942, and NGC 4631) are in galaxies in which strong dust lanes could have easily obscured any extant cluster. The other two nondetections (NGC 55 and IC 2233) are in low-mass galaxies without strong dust lanes, suggesting that these galaxies truly lack nuclear clusters.

In summary, we have detected candidate nuclear clusters in $50 \%-65 \%$ of our sample. Eliminating the galaxies with strong dust lanes, the fraction of galaxies with detected nuclear clusters may be as high as $82 \%$. These detection fractions are consistent with other studies, both for late-type galaxies, in which Böker et al. (2002) find that $\sim 75 \%$ of systems have clusters, and for early-type galaxies observed as part of the ACS Virgo Cluster Survey (Côté et al. 2006). However, our sample is less well-suited than these studies for constraining the frequency of nuclear clusters, because the edge-on perspective can result in more chance superpositions and greater obscuration from dust.

\subsection{Apache Point Observatory $3.5 \mathrm{~m}$ Spectrum}

To better constrain the stellar populations of the NGC 4244 nuclear cluster (the nearest in our sample), we obtained long-slit spectra on 2005 May 19 with the Double Imaging Spectrograph (DIS) on the Apache Point Observatory (APO) $3.5 \mathrm{~m}$ telescope. The DIS obtains both blue and red spectra simultaneously. We used the "medium-resolution" blue grating $\left(1.22 \AA\right.$ pixel $\left.^{-1}\right)$ and the "high-resolution" red grating $\left(0.84 \AA \mathrm{pixel}^{-1}\right)$. The resulting images had good-quality spectral coverage from 3700 to $5500 \AA$ on the blue side and 5600 to $7200 \AA$ on the red side. A 1".5 slit was used. Two exposures of 15 and 30 minutes were obtained, centered on the nuclear cluster and oriented along the major axis of NGC 4244 , with a position angle of $-43^{\circ}$. We note that the elongation of the nuclear cluster is aligned to within $\sim 10^{\circ}$ of the galaxy's major axis (see Fig. 1). Seeing at the time of observation was $\sim 1$." 0 , and thus the cluster was essentially unresolved. We reduced these spectra using a PyRAF pipeline designed for DIS data reduction (written by $\mathrm{K}$. Covey) that uses standard IRAF onedspec and twodspec routines. The NGC 4244 cluster observations were wavelength-calibrated using lamp spectra, and a further correction was applied to remove small shifts visible in the sky lines. The velocities were calibrated to the local standard of rest using rvcorrect and dopcor. Observations of Feige 66 at nearly identical air mass were obtained for flux calibration shortly before the observations; however, the night was not photometric, and thus the absolute flux scale is not fully constrained. The two observations of the NGC 4244 cluster differ in flux scale by under $10 \%$, and our absolute flux is probably uncertain by a similar amount. Combined, the two spectra give a signal-to-noise ratio $(\mathrm{S} / \mathrm{N})$ of $\sim 35 \mathrm{pixel}^{-1}$. The cluster spectrum was extracted from within a $2^{\prime \prime}$ diameter aperture, and regions used to define the background subtraction were within the confines of the galaxy, 
TABLE 1

Galaxy Sample and Nuclear Cluster Properties

\begin{tabular}{|c|c|c|c|c|c|c|c|c|}
\hline $\begin{array}{l}\text { Galaxy } \\
\text { (1) }\end{array}$ & $\begin{array}{l}\text { Type } \\
(2)\end{array}$ & $\begin{array}{l}M_{B} \\
\text { (3) }\end{array}$ & $\begin{array}{c}V_{\max } \\
\left(\mathrm{km} \mathrm{s}^{-1}\right) \\
(4)\end{array}$ & $\begin{array}{c}\text { Possesses Nuclear } \\
\text { Cluster? } \\
\text { (5) }\end{array}$ & $\begin{array}{c}\text { Multiple } \\
\text { Components? } \\
\text { (6) }\end{array}$ & $\begin{array}{l}M_{\mathrm{F} 814 \mathrm{~W}} \\
\quad(7)\end{array}$ & $\begin{array}{c}m_{\mathrm{F} 606 \mathrm{~W}}-m_{\mathrm{F} 814 \mathrm{~W}} \\
\text { (8) }\end{array}$ & $\begin{array}{c}m-M \\
\quad(9)\end{array}$ \\
\hline IC $2233 \ldots \ldots \ldots \ldots \ldots$ & SBcd & -16.60 & 79 & No & $\mathrm{N} / \mathrm{A}$ & $\ldots$ & $\ldots$ & $29.96^{\mathrm{a}}$ \\
\hline 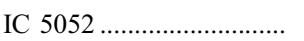 & SBcd & -17.23 & 79 & Yes & Yes & -10.77 & 1.08 & $28.90^{\mathrm{b}}$ \\
\hline IRAS 06070-6147 .................... & $\mathrm{Sc}$ & -20.50 & 133 & No; dust & $\mathrm{N} / \mathrm{A}$ & $\ldots$ & $\ldots$ & $31.22^{\mathrm{c}}$ \\
\hline IRAS $07568-4942 \ldots \ldots \ldots . . . .$. & $\mathrm{SBc}$ & -17.70 & 142 & No; dust & $\mathrm{N} / \mathrm{A}$ & $\cdots$ & $\cdots$ & $30.06^{\mathrm{d}}$ \\
\hline IRAS $09312-3248 \ldots \ldots \ldots$ & $\mathrm{Sc}$ & -17.90 & 98 & Maybe & No & -9.24 & 1.36 & $30.61^{\mathrm{c}}$ \\
\hline 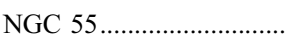 & $\mathrm{SBm}$ & -17.05 & 67 & No & N/A & $\ldots$ & $\ldots$ & $26.63^{\mathrm{b}}$ \\
\hline 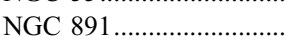 & $\mathrm{Sb}$ & -18.78 & 214 & $\mathrm{~N} / \mathrm{A}$ & $\mathrm{N} / \mathrm{A}$ & $\ldots$ & $\ldots$ & $29.61^{\mathrm{e}}$ \\
\hline 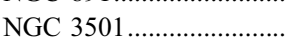 & $\mathrm{Sc}$ & -17.37 & 136 & Yes & No & -10.58 & 1.62 & $31.04^{\mathrm{c}}$ \\
\hline 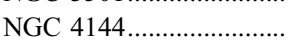 & $\mathrm{SBc}$ & -17.20 & 67 & Maybe & No & -8.33 & 0.85 & $29.36^{\mathrm{b}}$ \\
\hline 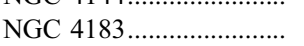 & $\mathrm{Sc}$ & -18.41 & 103 & Yes & No & -11.15 & 0.93 & $31.35^{\mathrm{f}}$ \\
\hline 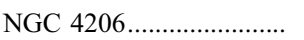 & $\mathrm{Sbc}$ & -18.46 & 124 & Yes & Yes & -14.17 & 0.93 & $31.29^{\mathrm{g}}$ \\
\hline 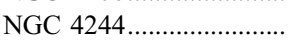 & $\mathrm{Sc}$ & -17.08 & 93 & Yes & Yes & $<-12.65^{\mathrm{h}}$ & 0.77 & $28.20^{\mathrm{b}}$ \\
\hline 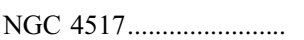 & $\mathrm{Sc}$ & -18.18 & 137 & Yes & Maybe & -11.34 & 1.77 & $29.30^{\mathrm{d}}$ \\
\hline 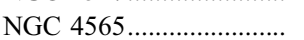 & $\mathrm{Sb}$ & -20.47 & 227 & N/A & N/A & $\ldots$ & $\ldots$ & $31.05^{\mathrm{i}}$ \\
\hline 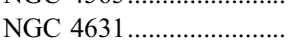 & SBcd & -19.75 & 131 & No; dust & N/A & $\cdots$ & $\cdots$ & $29.42^{\mathrm{b}}$ \\
\hline 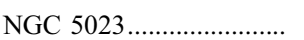 & $\mathrm{Sc}$ & -16.29 & 77 & Yes & Maybe & -9.79 & 1.26 & $29.10^{\mathrm{b}}$ \\
\hline
\end{tabular}

Note.-Types and velocities are from HYPERLEDA/LEDA (Paturel et al. 1995, 2003).

${ }^{\text {a }}$ Bottinelli et al. (1985).

b Paper I.

c Assuming $H_{0}=70 \mathrm{~km} \mathrm{~s}^{-1} \mathrm{Mpc}^{-1}$.

d Bottinelli et al. (1988).

e Tonry et al. (2001).

f Tully \& Pierce (2000).

g Gavazzi et al. (1999).

$\mathrm{h}$ The derived magnitudes on the NGC 4244 cluster are lower limits due to the central pixels being saturated in both bands.

i Jensen et al. (2003).

$20^{\prime \prime}-30^{\prime \prime}$ (approximately one-fourth the scale length of the galaxy) along the major axis in both directions. Any contamination from the underlying galaxy component should be very small, as the source was well above the background at all wavelengths. We analyze these data in $\S \S 4$ and 5 .

\section{NUCLEAR CLUSTER MORPHOLOGIES AND LUMINOSITIES}

The most basic result of this paper is that nuclear clusters are not all simple, single-component objects, as is evident from inspection of Figure 1, which shows the F814W images of all nine nuclear cluster candidates. Of these candidates, three (IC 5052, NGC 4206, and NGC 4244) look like miniature S0 galaxies, possessing both an elongated disk or ring component and a spheroidal component. These components are both compact with physical dimensions of tens of parsecs or less. We show in $\S 3.1 .3$ that these components appear to physically overlap. We therefore refer to these clusters as "multicomponent clusters" throughout the rest of the paper. Two other galaxies, NGC 4517 and NGC 5023, also show tentative evidence for similar structures. In this section we focus on modeling the morphology of the clusters, in particular those that have multiple components. We show that (1) the nuclear cluster elongations are closely aligned with the disk of the host galaxy, (2) models with both a spheroidal and disk or ring components fit the multicomponent clusters significantly better than single-component models, and (3) the nuclear clusters in our sample have magnitudes and sizes similar to those observed in other galaxies.

\subsection{Model Fitting}

The dimensions and scales of the clusters were determined via two-dimensional fitting to analytical functions (e.g., King profiles). Because the objects are very compact, it was necessary to include the effects of the point-spread function (PSF). We used two similar methods to fit the nuclear clusters. First, we used the ishape program (Larsen 1999), which was designed to fit semiresolved clusters. Unfortunately, ishape only works with elliptically symmetric objects, which some of our objects clearly are not. Therefore, we wrote our own code in IDL, using the existing MPFIT2DFUN package to perform Levenberg-Marquardt leastsquares fits.

In both ishape and our program, analytical functions are convolved with the PSF before fitting to the data. We performed this convolution using a position-variable PSF derived from our data (see Paper I for details), and we oversampled by a factor of 10 to minimize the effects of aliasing and binning. A separate PSF was generated for each cluster based on its position on the chip. We conducted tests to ensure that our program gave results similar to those of the ishape program.

We used ishape (1) to determine what types of functions best fit the data and (2) to assess whether each cluster was indeed resolved. We fit Gaussian, King, Moffat, and Hubble profiles to each cluster and found that King and Moffat profiles provided the best overall fits in terms of the reduced $\chi^{2}$, as also found by Böker et al. (2004). Both profiles provided equally good fits to our clusters, and we chose to continue our fitting with elliptical King profiles of the form

$$
\begin{gathered}
\Sigma(z)=\Sigma_{0}\left[\frac{1}{\sqrt{1+\left(z / r_{\text {core }}\right)^{2}}}-\frac{1}{\sqrt{1+\left(r_{\text {tidal }} / r_{\text {core }}\right)^{2}}}\right] \\
\text { with } z=\sqrt{x^{2}+(z / q)^{2}},
\end{gathered}
$$

where $\Sigma(z)$ is the projected surface brightness, $x$ and $z$ are the coordinates along the major and minor axes, and $q$ is the axial 

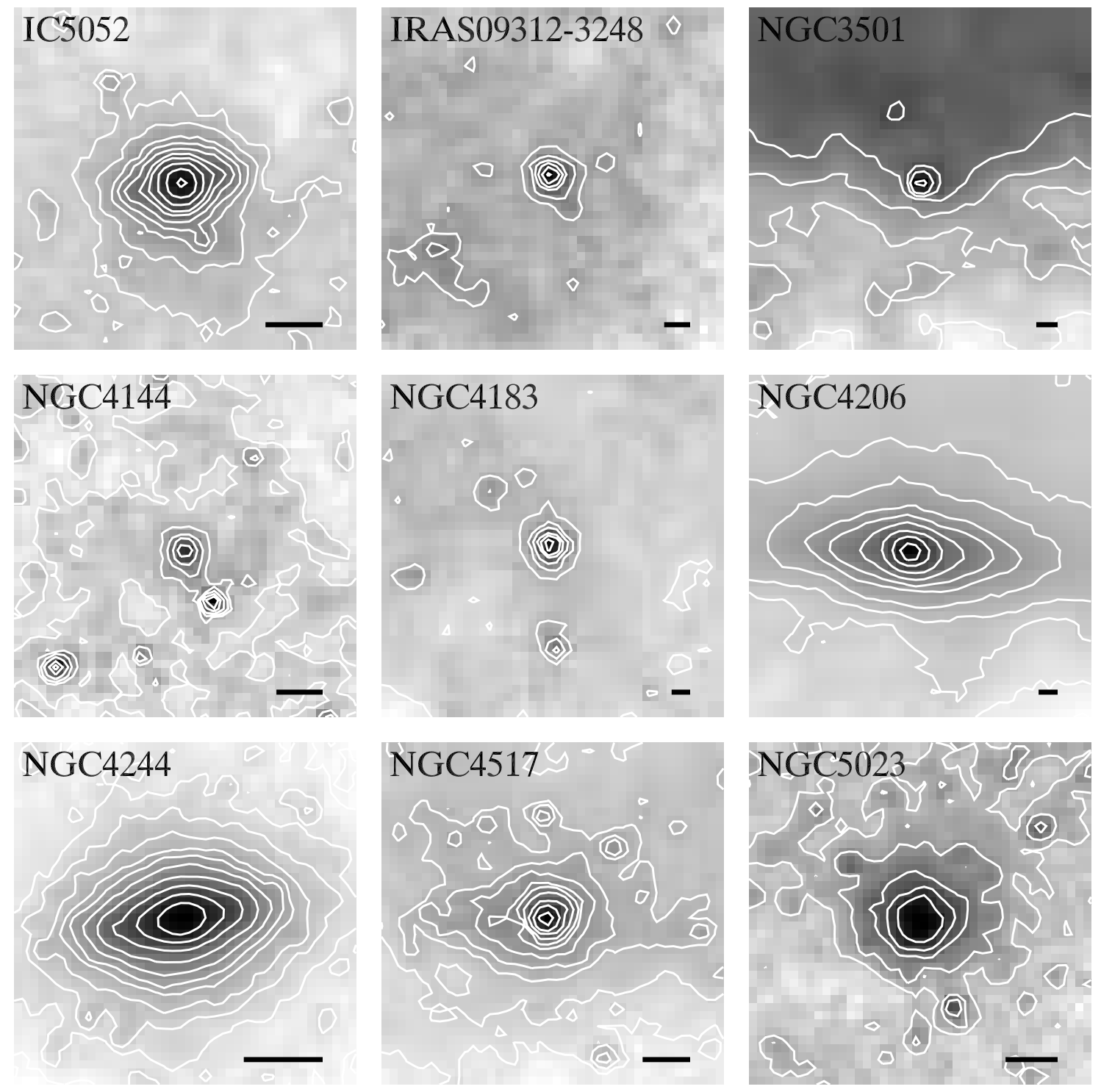

FIG. 1.- Images in the F814W filter of the nine candidate nuclear clusters. Each image is $2^{\prime \prime}$ on a side, and the bar in the bottom right corner indicates a length of $10 \mathrm{pc}$. Images have been rotated so that the $x$-axis lies along the position angle of the major axis of the galaxy disk. [See the electronic edition of the Journal for a color version of this figure.]

ratio (minor/major). We have fixed the concentration $(c \equiv$ $\left.r_{\text {tidal }} / r_{\text {core }}\right)$ to be 15 for our fits, based on the fit concentrations of our brightest clusters and similar to that found for Galactic analog $\omega$ Cen (Trager et al. 1995). We report only half-light (effective) radii, as this quantity is well determined even for relatively low S/N data (Carlson \& Holtzman 2001) and does not depend strongly on the chosen model (see Fig. 2 of Böker et al. 2004).

Given sufficient $\mathrm{S} / \mathrm{N}$, semiextended sources can be detected with intrinsic widths as small as $10 \%$ of the PSF (Larsen 1999). The FWHM of our PSF is $\sim 0.9$ pixels, meaning objects with widths smaller than 1 pixel should be easily resolvable. The $\mathrm{S} / \mathrm{N}$ of our fits ranged from $\sim 50$ to 1500 . For four of the galaxies (IC 5052, NGC 4206, NGC 4244, and NGC 5023), the FWHM of the fitted ishape King profiles is greater than 1 pixel, and the objects are clearly partially resolved. The $\chi^{2}$ values of these fits are 2-20 times better than the $\chi^{2}$ of a point-source fit (see last column of Table 2). The remaining fits have FWHM $>0.2$ pixels and $\chi^{2}$ values 1-3 times the best-fitting point-source $\chi^{2}$. Of these,
NGC 4144 and NGC 4517 are still clearly resolved, while IRAS 09312-3248, NGC 3501, and NGC 4183 (all of which are at large distances) are only marginally resolved.

\subsubsection{Elliptical King Model Fits}

Table 2 shows the results of our program's fits to singlecomponent elliptical King profiles with $c=15$. These fits show the following:

1. All the well-resolved clusters are flattened, as shown in Figure 2. The median axis ratio $(q \equiv b / a)$ is 0.81 , with $q \sim 0.4$ for NGC 4206 and NGC 4244, the two systems with the most prominent disks (see $\S 3.1 .2$ ).

2. The position angles of the three multicomponent nuclear clusters (IC 5052, NGC 4206, and NGC 4244) are all aligned within $10^{\circ}$ of the galaxy disk position angle. This can be seen plainly in Figures 1 and 2. The NGC 4517 and NGC 5023 clusters also are somewhat elongated along the plane of the galaxy. 
TABLE 2

Elliptical King Profile Fits

\begin{tabular}{|c|c|c|c|c|c|c|c|}
\hline Galaxy & $\begin{array}{l}\text { Pixel Scale } \\
\left(\mathrm{pc} \mathrm{pixel}^{-1}\right)\end{array}$ & Filter & $\begin{array}{l}r_{\text {eff }} \\
(\mathrm{pc})\end{array}$ & $q(b / a)$ & $\begin{array}{l}\Delta \text { P.A. }^{\mathrm{a}} \\
(\mathrm{deg})\end{array}$ & Reduced $\chi^{2}$ & $\chi_{\mathrm{ps}}^{2} / \chi^{2}$ (ishape) \\
\hline IC 5052 & 1.46 & F606W & 3.64 & 0.76 & -3.8 & 23.8 & 5.78 \\
\hline IC 5052 & 1.46 & F814W & 2.93 & 0.80 & -6.6 & 18.1 & 7.00 \\
\hline IRAS $09312-3248 \ldots \ldots \ldots \ldots \ldots . . .$. & 3.21 & F606W & 2.46 & 0.68 & 43.1 & 2.8 & 1.50 \\
\hline 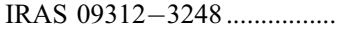 & 3.21 & F814W & 2.24 & 0.86 & 39.8 & 3.5 & 1.42 \\
\hline 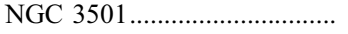 & 3.91 & F606W & 6.82 & 1.00 & 89.9 & 8.2 & 1.41 \\
\hline 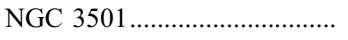 & 3.91 & F814W & 3.58 & 0.61 & 19.2 & 10.1 & 1.38 \\
\hline 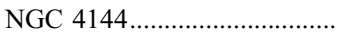 & 1.81 & F606W & 1.46 & 0.85 & 83.4 & 3.2 & 2.69 \\
\hline 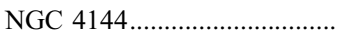 & 1.81 & F814W & 1.99 & 0.81 & 74.8 & 4.6 & 2.30 \\
\hline 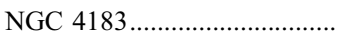 & 4.51 & F606W & 2.76 & 1.00 & -40.8 & 9.6 & 1.20 \\
\hline 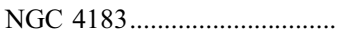 & 4.51 & F814W & 3.02 & 0.78 & 68.8 & 6.4 & 1.12 \\
\hline 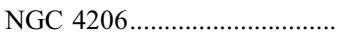 & 4.39 & F606W & 16.30 & 0.37 & 5.6 & 54.7 & 2.39 \\
\hline 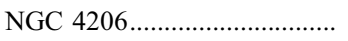 & 4.39 & F814W & 15.81 & 0.40 & 5.3 & 42.4 & 2.75 \\
\hline NGC 4244................................ & 1.06 & F606W & 2.92 & 0.46 & -10.0 & 94.1 & 20.73 \\
\hline NGC 4244 ................................. & 1.06 & F814W & 3.35 & 0.49 & -9.9 & 82.3 & 19.63 \\
\hline 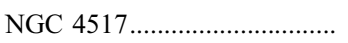 & 1.76 & F606W & 1.71 & 0.85 & -22.9 & 46.0 & 1.20 \\
\hline NGC 4517................................. & 1.76 & F814W & 1.33 & 0.85 & -16.1 & 64.2 & 1.23 \\
\hline 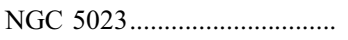 & 1.60 & F606W & 12.54 & 0.81 & 16.2 & 13.4 & 4.27 \\
\hline 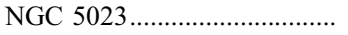 & 1.60 & F814W & 10.22 & 0.83 & 18.6 & 11.1 & 6.89 \\
\hline
\end{tabular}

a $\Delta$ P.A. is the position angle relative to the galaxy disk.

3. The effective radius (also known as the half-light radius) ranges between 1 and $20 \mathrm{pc}$, with most of the clusters having effective radii between 1 and $4 \mathrm{pc}$. Figure 3 demonstrates that the measured effective radii are similar to those observed for other objects, including late-type nuclear clusters ( filled circles; Böker et al. 2004) and Galactic globular clusters (open triangles; Harris 1996).

4. After subtraction of the King profile fit, the residuals of IC 5052, NGC 4206, and NGC 4244 clearly show evidence for a

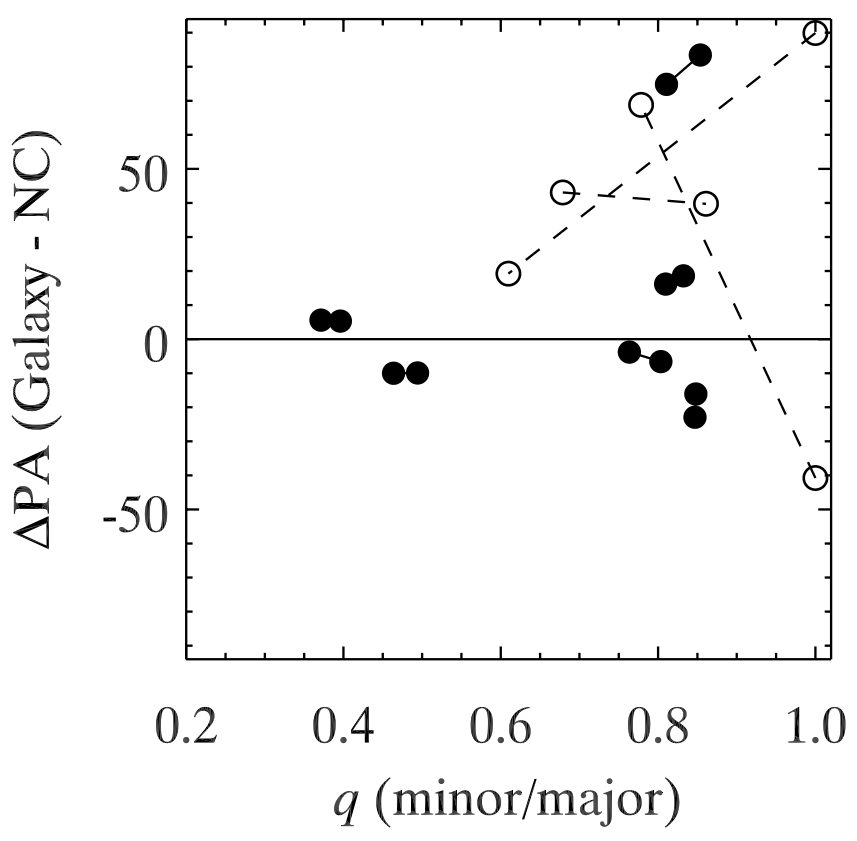

FIG. 2.-Elliptical King profile fit results. The axial ratio $q$ is plotted against the position angle of the major axis of the nuclear cluster relative to the galaxy disk ( $\Delta$ P.A.). The connected data points connect the fits for each galaxy in the two filters. Open circles indicate the three galaxies (IRAS 09312-3248, NGC 3501 , and NGC 4183) for which good fits were not achieved due to the nuclear cluster compactness and complexity of the surrounding emission. This figure shows that most of the clusters we have detected were significantly flattened, and that the flattening aligns closely with the plane of the galaxy.

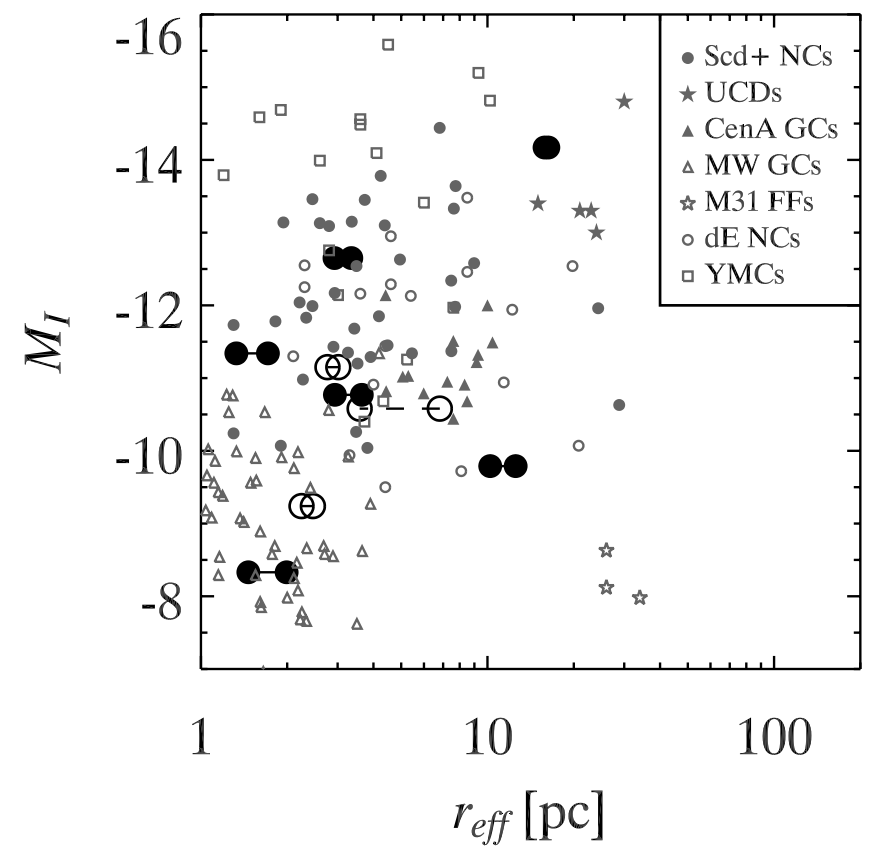

FIG. 3.-Fitted effective radius vs. absolute aperture magnitude in the F814W filter. Large circles are the same as in Fig. 2. Small symbols denote the following: filled circles, late-type nuclear clusters from Böker et al. (2004); open circles, dE,N nuclei from De Propris et al. (2005) (transformed to $M_{I}$ using Padova models, which suggests that stellar populations older than 1 Gyr have F814W F850LP colors of $0.25 \pm 0.1 \mathrm{mag}$ ); filled triangles, massive globular clusters in NGC 5128 observed by Martini \& Ho (2004); open triangles, Galactic globular clusters from the Harris (1996) catalog (note that $\omega$ Cen is the brightest of these); filled stars, Fornax UCDs from Drinkwater et al. (2003), assuming $V-I$ of $1.1 \pm 0.1$ as shown in Mieske et al. (2002); open stars, faint fuzzy M31 clusters from Huxor et al. (2005); open squares, young massive clusters from Bastian et al. (2006). Note that Padova models suggest $M_{\mathrm{F} 814 \mathrm{~W}} \sim M_{I}$ to within a few hundredths of a magnitude. [See the electronic edition of the Journal for a color version of this figure.] 


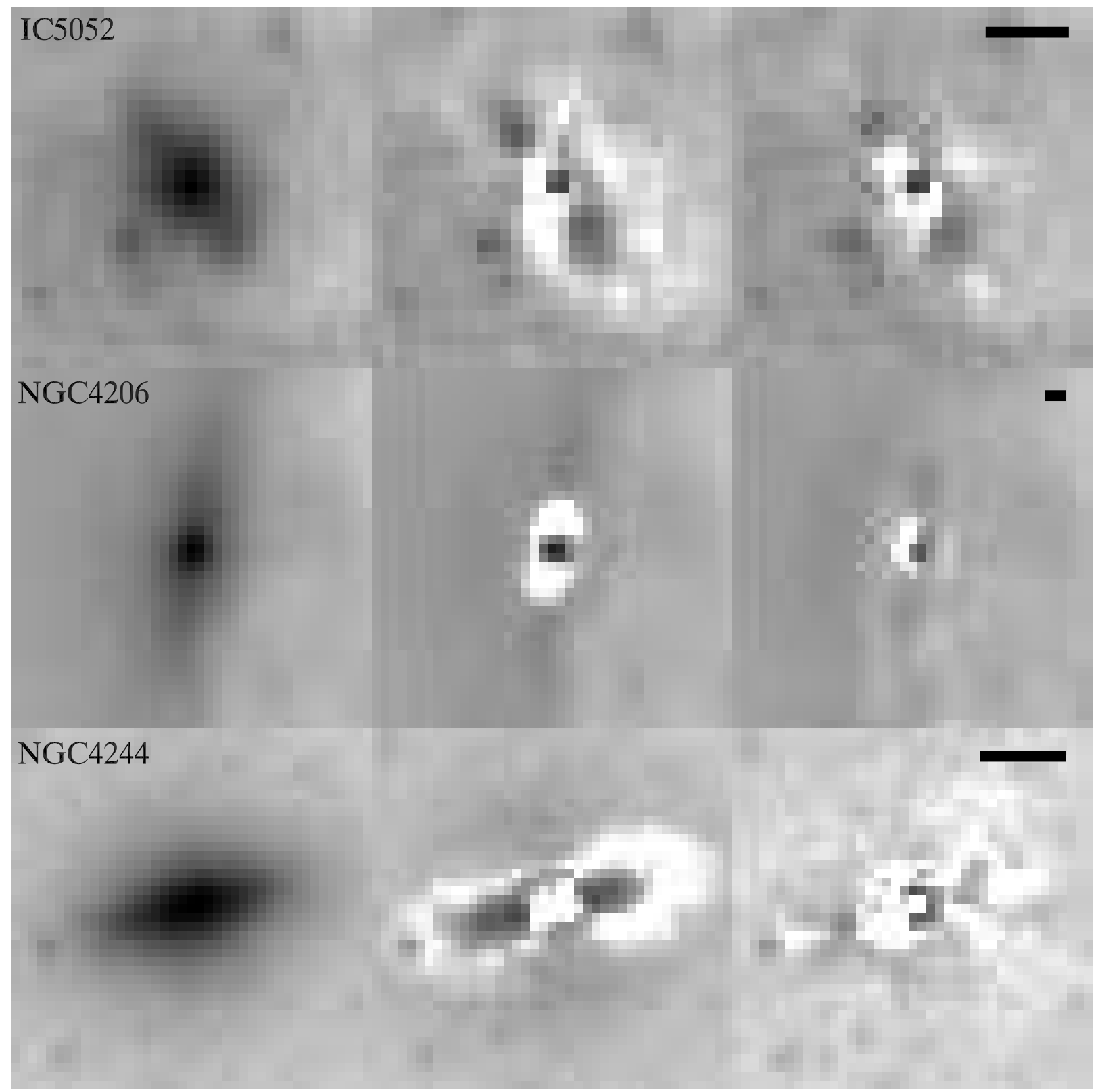

FIG. 4.- Residuals of model fits to F606W observations of nuclear clusters in IC 5052, NGC 4206, and NGC 4244. The images show the area over which fits were done and are in the coordinate system of the ACS images. They are thus rotated from the images in Figs. 1 and 5 . Bars in the upper right corner show a length of $10 \mathrm{pc}$ in each galaxy. The left column shows the original images, and the middle and right columns show the images after subtraction of the best-fitting elliptical King and elliptical King + disk profiles. All are shown with the same scaling. In each case the elliptical King + disk profile clearly provides a better fit to the cluster than a single-component elliptical King profile.

flat disklike or ringlike component (Fig. 4, middle column). The reduced $\chi^{2}$ of these three galaxies is also very high, suggesting that the single-component King profile is not a good fit to the data. We note that NGC 4517, which has a disklike appearance in its outer isophotes, also has a high $\chi^{2}$ value. In NGC 4244 the central 8 pixels were saturated and therefore excluded from the fit.

\subsubsection{Elliptical King + Disk Models}

To account for the flattened residuals remaining after fits to the elliptical King profiles in the IC 5052, NGC 4206, and NGC 4244 nuclear clusters, we have fit an additional morphological component to these clusters. In this section we examine fits to disk models; in $\S 3.1 .3$ we consider fits to ring models and compare the disk and ring fits.
As a disk model we adopted the surface brightness profile of a transparent, self-gravitating, edge-on exponential disk (van der Kruit \& Searle 1981),

$$
\Sigma(x, z)=\Sigma_{0} \frac{x}{h_{x}} K_{1}\left(\frac{x}{h_{x}}\right) \operatorname{sech}^{2}\left(\frac{z}{z_{0}}\right),
$$

where $x$ and $z$ are the major and minor axis coordinates, $\Sigma_{0}$ is the central surface brightness, $K_{1}$ is the modified Bessel function, $h_{x}$ is the disk's exponential scale length, and $z_{0}$ is the scale height of the disk. We note that we tried other vertical profile fits (e.g., simple exponential) that were too concentrated near the midplane to provide good fits to the data.

Figure 4 shows that the inclusion of a disk component clearly improves the fit relative to the single-component elliptical King 
TABLE 3

Elliptical King + Disk Profile Fits

\begin{tabular}{|c|c|c|c|c|c|c|c|c|c|c|}
\hline \multirow[b]{2}{*}{ GALAXY } & \multirow[b]{2}{*}{$\begin{array}{l}\text { PIXEL SCALE } \\
\left(\mathrm{pc} \mathrm{pixel}^{-1}\right)\end{array}$} & \multirow[b]{2}{*}{ FILTER } & \multicolumn{3}{|c|}{ DISK } & \multicolumn{3}{|c|}{ ElLIPTICAL KING } & \multirow[b]{2}{*}{$\begin{array}{l}\Delta \text { P.A. } \\
(\mathrm{deg})\end{array}$} & \multirow[b]{2}{*}{ REDUCED $\chi^{2}$} \\
\hline & & & $\begin{array}{c}\mu_{0} \\
\left(\mathrm{mag} \operatorname{arcsec}^{-2}\right)\end{array}$ & $\begin{array}{c}h_{x} \\
(\mathrm{pc})\end{array}$ & $\begin{array}{c}z_{0} \\
(\mathrm{pc})\end{array}$ & $\begin{array}{c}\mu_{0} \\
\left(\operatorname{mag} \operatorname{arcsec}^{-2}\right)\end{array}$ & $\begin{array}{l}r_{\mathrm{eff}} \\
(\mathrm{pc})\end{array}$ & $q$ & & \\
\hline IC $5052 \ldots \ldots \ldots \ldots \ldots \ldots$ & 1.46 & F606W & 17.27 & 3.54 & 1.47 & 16.15 & 3.97 & 1.00 & -10.8 & 20.3 \\
\hline 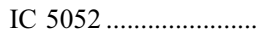 & 1.46 & F814W & 16.28 & 3.48 & 1.19 & 14.74 & 3.55 & 1.00 & -12.0 & 15.7 \\
\hline NGC $4206 \ldots \ldots \ldots \ldots . . . . . . . .$. & 4.39 & F606W & 17.44 & 31.00 & 15.76 & 12.87 & 4.94 & 0.45 & 5.1 & 26.8 \\
\hline 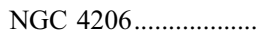 & 4.39 & F814W & 16.61 & 31.36 & 17.42 & 12.00 & 5.32 & 0.44 & 5.0 & 16.7 \\
\hline 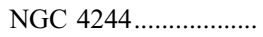 & 1.06 & F606W & 13.64 & 2.51 & 1.64 & 16.29 & 10.79 & 0.78 & -10.1 & 15.1 \\
\hline 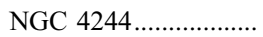 & 1.06 & F814W & 13.18 & 2.73 & 1.42 & 13.84 & 5.70 & 0.73 & -9.6 & 16.3 \\
\hline
\end{tabular}

profile. Quantitatively, the $\chi^{2}$ of the fits shown in Table 3 is significantly reduced relative to those for the single-component fit (Table 2). The improvement is most dramatic in NGC 4244 and is least significant in IC 5052. However, the reduced $\chi^{2}$ values remain well above unity, suggesting the model does not fit the data perfectly. For IC 5052 and NGC 4244, the disk scale lengths are comparable to the effective radii of the spheroidal components $\left(h_{x} \sim 3 \mathrm{pc}\right)$, while in NGC 4206 the disk component is very extended $\left(h_{x}=31 \mathrm{pc}\right)$.

In NGC 4206 and IC 5052, the residuals show a similar pattern that suggests the presence of a compact central source and/or a ring rather than a disk. We discuss the issue of whether the components are rings or disks in $\S 3.1 .3$. Both these galaxies also show a color gradient perpendicular to the major axis, suggesting the presence of a thin dust disk.

\subsubsection{Rings versus Disks}

In this section we present evidence that the flattened components in the NGC 4206 and NGC 4244 clusters are better fit by a disk model than a ring model, while in IC 5052 a ring model provides a better fit. In all three cases it appears that the disk or ring extends to small radii and overlaps with the light distribution of the spheroidal component.

In Table 4 we present the best-fitting ring + elliptical King models for each image. For the ring component, we assumed a constant midplane density with an outer $\left(r_{\text {out }}\right)$ and inner $\left(r_{\text {in }}\right)$ radius,

$$
\begin{aligned}
& \Sigma(x, z)= \\
& \begin{cases}2 \rho_{0} \operatorname{sech}^{2}\left(\frac{z}{z_{0}}\right)\left(\sqrt{r_{\text {out }}^{2}-x^{2}}-\sqrt{r_{\text {in }}^{2}-x^{2}}\right), & x<r_{\text {in }}, \\
2 \rho_{0} \operatorname{sech}^{2}\left(\frac{z}{z_{0}}\right)\left(\sqrt{r_{\text {out }}^{2}-x^{2}}\right), & r_{\text {in }}<x<r_{\text {out }}, \\
0, & \text { else. }\end{cases}
\end{aligned}
$$

This model is brightest for $r_{\text {in }}<x<r_{\text {out }}$ and contributes less flux near the center, making it qualitatively different from the disk model (eq. [2]). However, given that the details of both models are somewhat arbitrary, the results of the fits should be viewed with some caution.

For the NGC 4206 and NGC 4244 nuclear clusters, the ring fits (Table 4) have reduced $\chi^{2}$ values that are $20 \%-75 \%$ higher than the disk values shown in Table 3 . Furthermore, the best-fitting ring models have inner radii extending all the way to the center of the cluster. Thus, the evidence suggests that in the NGC 4206 and NGC 4244 nuclear clusters, the second, flattened component is disklike rather than ringlike.

In contrast, the nuclear cluster in IC 5052 shows two symmetric spots in its residuals (Fig. 4) and color map (Fig. 5). Not surprisingly, the ring model provided a better fit ( $20 \%$ decrease in reduced $\chi^{2}$ ), with an inner and outer radius of the ring of 6.8 and $11.8 \mathrm{pc}$, respectively. The whole ring falls well within the tidal radius of the spheroidal component $(\sim 24 \mathrm{pc})$, and thus their stellar distributions appear to overlap.

Nuclear rings have previously been detected in molecular gas (e.g., Böker et al. 1997, 1998) and UV stellar light (e.g., Maoz et al. 1996). However, these studies find rings an order of magnitude larger in size than the one we find in IC 5052. The only previously observed structure around a nuclear cluster on a scale similar to that of the IC 5052 ring and NGC 4244 disk is the compact molecular disk detected coincident with the nuclear cluster in IC 342 (Schinnerer et al. 2003), which has a diameter of 30$55 \mathrm{pc}$, depending on the assumed distance to the galaxy. We note that this disk is barely resolved by their $\mathrm{CO}$ observations (with a resolution of 1 ".2) and may therefore be either a disk or a ring.

The evidence in all three clusters that the disk or ring components do overlap with the spheroidal components justifies our labeling these three nuclear clusters as multicomponent clusters. For simplicity, and because disk models are preferred in two of the three multicomponent clusters, we refer to the flattened

TABLE 4

\begin{tabular}{|c|c|c|c|c|c|c|c|c|c|c|}
\hline \multirow[b]{2}{*}{ GaLAXY } & \multirow[b]{2}{*}{ Filter } & \multicolumn{4}{|c|}{ RING } & \multicolumn{3}{|c|}{ ElLiptical King } & \multirow[b]{2}{*}{$\begin{array}{l}\Delta \text { P.A. } \\
\text { (deg) }\end{array}$} & \multirow[b]{2}{*}{ Reduced $\chi^{2}$} \\
\hline & & $\begin{array}{l}r_{\text {out }} \\
(\mathrm{pc})\end{array}$ & $\begin{array}{c}r_{\text {in }} \\
(\mathrm{pc})\end{array}$ & $\begin{array}{c}z_{0} \\
(\mathrm{pc})\end{array}$ & $L_{\text {ring }} / L_{\text {tot }}$ & $\begin{array}{c}\mu_{0} \\
\text { (mag } \operatorname{arcsec}-2)\end{array}$ & $\begin{array}{r}r_{\text {eff }} \\
(\mathrm{pc})\end{array}$ & $q$ & & \\
\hline 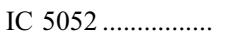 & F606W & 11.83 & 6.83 & 1.44 & 0.18 & 15.64 & 3.24 & 1.00 & -11.0 & 16.9 \\
\hline 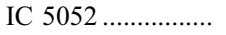 & F814W & 12.13 & 6.91 & 1.61 & 0.14 & 14.36 & 3.05 & 1.00 & -11.5 & 13.2 \\
\hline NGC 4206............ & F606W & 84.82 & 0.44 & 18.56 & 0.53 & 13.57 & 7.69 & 0.48 & 5.3 & 32.5 \\
\hline NGC 4206.............. & F814W & 83.78 & 0.44 & 20.69 & 0.53 & 12.59 & 7.70 & 0.47 & 5.2 & 21.5 \\
\hline NGC $4244 \ldots \ldots \ldots \ldots . . . .$. & F606W & 10.42 & 0.11 & 0.92 & 0.18 & 13.19 & 4.07 & 0.58 & -10.1 & 26.7 \\
\hline 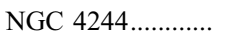 & F814W & 10.75 & 0.14 & 0.91 & 0.16 & 12.51 & 4.25 & 0.59 & -9.7 & 23.5 \\
\hline
\end{tabular}

Elliptical King + Ring Profile Fits 

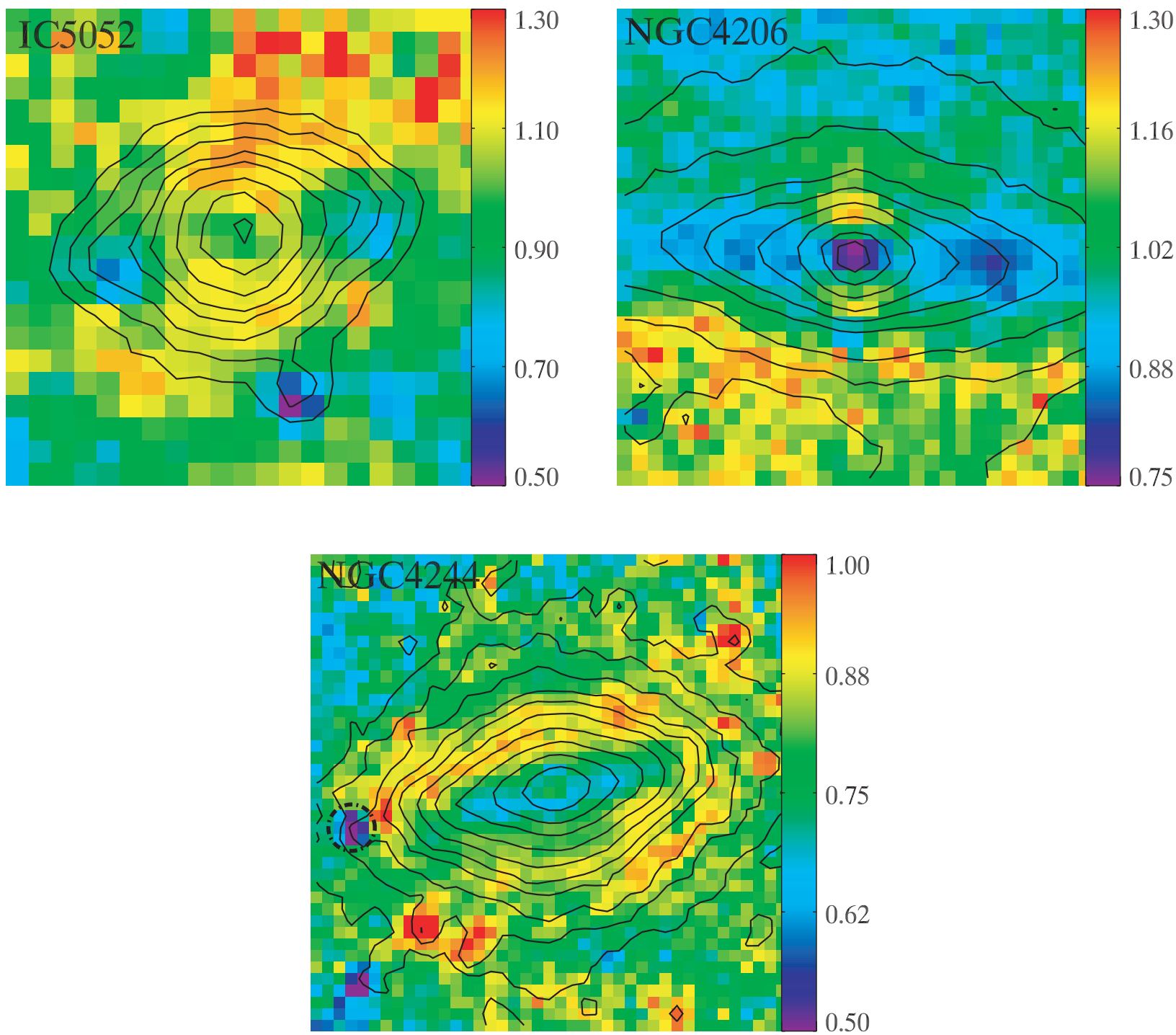

Fig. 5.- Maps of the F606W - F814W (VEGAMAG) color for each cluster. Contours showing the F606W brightness are overlaid in black. The clusters with obvious disk components (IC 5052, NGC 4206, and NGC 4244) all show bluer disks and redder spheroids. The color bars on the right side indicate values for the F606W - F814W color. Colors have been corrected for foreground reddening. The central 8 pixels of the NGC 4244 nuclear cluster were saturated and thus do not give accurate colors. The circled region in NGC 4244 shows the suspected H II region used to obtain a dynamical mass estimate in $\S 5$.

components in the next few sections as disks. We return to the formation and possible role of rings in our discussion (§ 7.1.3).

\subsection{Luminosities}

The F814W absolute magnitude $\left(M_{\mathrm{F} 814 \mathrm{~W}}\right)$ and the F606W F814W color are given in Table 1. Photometry was done using the Vega-based zero points and reddening coefficients from Sirianni et al. (2005). The foreground reddenings were corrected using values of $E(B-V)$ from Schlegel et al. (1998) that were at most 0.05 mag. These measurements are complicated by the high and variable background level and the complex shapes of the nuclear clusters. A scatter of $\sim 0.1 \mathrm{mag}$ was found by varying the aperture size within reasonable limits. The formal errors on the calculated magnitudes are quite small (typically $<0.01 \mathrm{mag}$ ). Magnitudes from the fitted models agree with the aperture magnitudes in cases in which the data are well fit, but they differ substantially when the fits are poor.

Figure 3 plots the F814W aperture magnitudes measured for all nine nuclear candidates against their fitted half-light radii from the elliptical King profiles. We also plot magnitudes and sizes of other compact stellar systems, assuming that $M_{I} \sim M_{\mathrm{F} 814 \mathrm{~W}}$, since the F $814 \mathrm{~W}$ filter is very similar to the Johnson $I$ filter, with single stellar populations expected to be $\sim 0.03$ mag brighter in $\mathrm{F} 814 \mathrm{~W}$ than in I (Girardi 2006).

Examination of Figure 3 shows that the magnitudes and sizes of the objects in our sample are similar to the nuclear clusters found by Böker et al. (2002, 2004; filled circles) for their sample of face-on, late-type galaxies. Böker et al. (2004) measure sizes for only the brighter clusters in their sample but find nuclear clusters with measured magnitudes as faint as $M_{I}=-8.6$. Because of their galaxies' face-on orientations, clusters with disk and spheroid components such as we have observed would have gone unnoticed in their study. The morphology of the M33 nuclear cluster is flattened $(\epsilon=0.17)$ along an axis parallel to the major axis of the galaxy (Lauer et al. 1998; Matthews et al. 1999), as would be expected for a multicomponent cluster observed at the inclination of M33's disk. The stellar populations in the spectra of both M33's nuclear cluster (Long et al. 2002) and some of the objects in the Böker et al. (2002) sample (Walcher et al. 2006) are also similar to what we see in $\S 4.2$ for NGC 4244's nuclear 


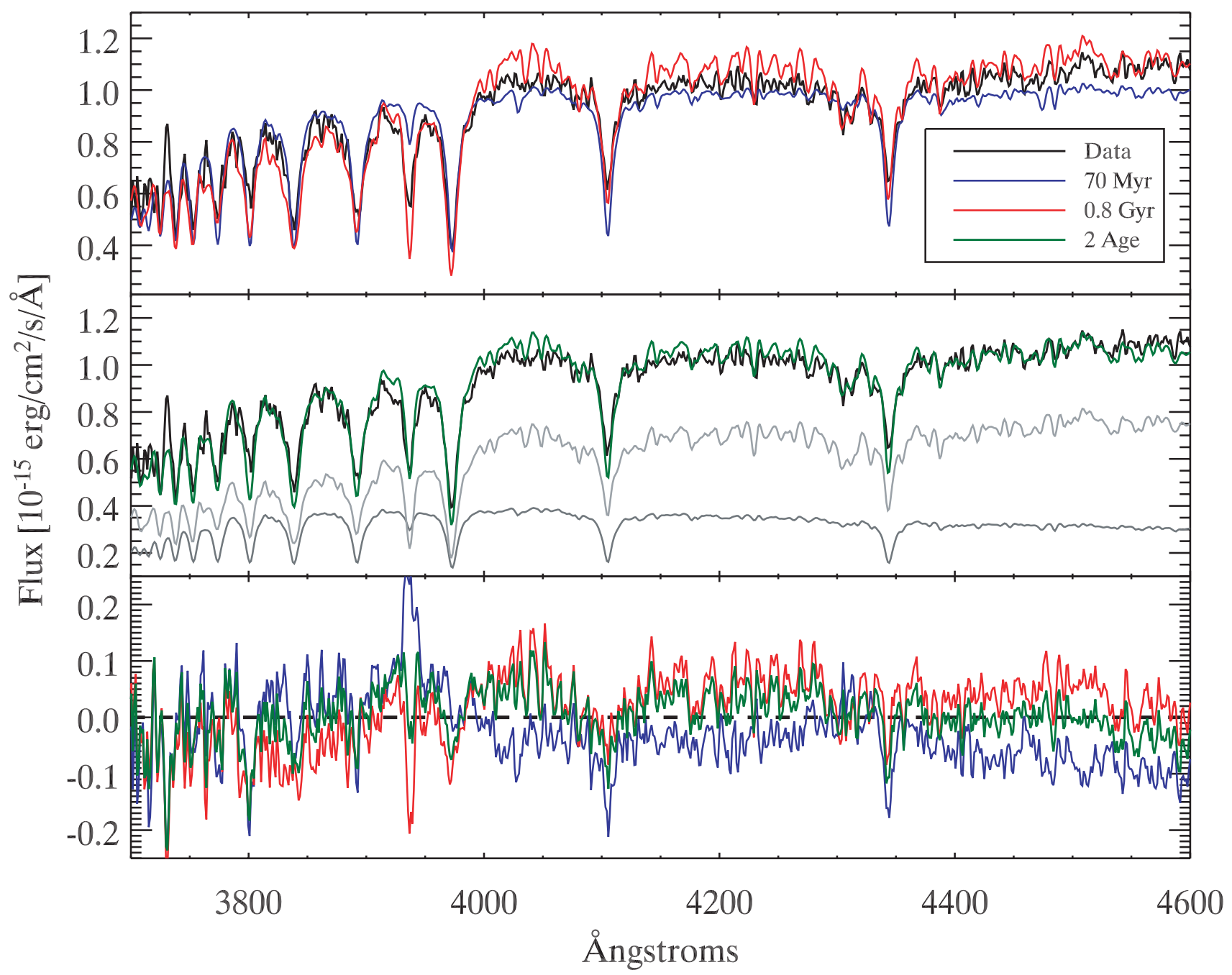

FIG. 6.-Portion of the APO DIS spectrum of the NGC 4244 nuclear cluster with best-fitting models overplotted. Only the portion of the spectrum with the most significant spectral features is shown. Top: Single-age best fits at $70 \mathrm{Myr}$ and $0.8 \mathrm{Gyr}$, the ages at which there are minima in the joint $\chi^{2}$ distribution (see Fig. 7). The most age-sensitive portion of the spectrum lies around the $\mathrm{Ca} \mathrm{H}$ line at $23940 \AA$, although numerous other features also cannot be fit well with a single-age population. Middle: Best two-age fit, including both stars with ages of $0.1 \mathrm{Gyr}$ (dark-gray line) and $1.0 \mathrm{Gyr}$ (light-gray line). This fit is a significant improvement relative to either single-age fit. Bottom: Residuals for all three fits.

cluster. It is therefore clear that the nuclear clusters observed here are similar to those found previously. Furthermore, the multicomponent morphology may be a common feature of nuclear clusters.

We also note that the two most luminous clusters are those with the most prominent disk components (NGC 4206 and NGC 4244). This correlation is not surprising, as the disks seem to be composed of recently formed stars (see $\S 4$ ) and would therefore have low mass-to-light ratios. Over time, as the young stellar populations age, the disks will become less prominent.

The observed color of the clusters (see Table 1) can constrain the total dust column to the center of the host galaxies. We estimated rough lower and upper limits on the total reddening by comparing the colors of the nuclear clusters to those of an old and red or a young and blue stellar population. For most of the galaxies, the F606W - F814W nuclear cluster colors of $\sim 1$ suggest that the reddening lies between $E(B-V)$ values of 0 and 1 . However, for NGC 3501 and NGC 4517 the reddening must be greater than $0.7 \mathrm{mag}$.

\section{NUCLEAR CLUSTER STELLAR POPULATIONS}

In this section we demonstrate that the multiple structural components seen in some nuclear clusters in $\S 3$ also have distinct stellar populations. In particular, the disk components are made of younger stars than the spheroidal components. We first examine color maps of the clusters and argue that the disks most likely have populations younger than $1 \mathrm{Gyr}$. We then examine the bright nearby nuclear cluster in NGC 4244 in detail, using multiband photometry and spectroscopy (see Fig. 6) to show that the spectra is best fit by two or more epochs of star formation.

Two recent papers have presented very thorough studies of the stellar populations of nuclear clusters in both early- and late-type spiral galaxies (Walcher et al. 2006; Rossa et al. 2006). Both use fitting of spectra to convincingly show that nuclear clusters contain multiple generations of stars. For a sample of nine bright nuclear clusters in late-type galaxies, Walcher et al. (2006) has shown that all of the clusters observed had stellar populations younger than $100 \mathrm{Myr}$. The typical mass of the youngest stars was a few $\times 10^{5} M_{\odot}$. However, in most of the clusters, the bulk of the stellar mass had an age of $\gtrsim 2.5$ Gyr. Our findings on the stellar populations in our multicomponent clusters, presented below, are fully consistent with these papers.

\subsection{Color Maps}

Figure 5 shows F606W - F814W color maps for the three nuclear cluster candidates identified as having multiple components. In each cluster, an obvious and significant color difference 
exists between the disk and spheroidal components. The disks are $0.3-0.6$ mag bluer in $\mathrm{F} 606 \mathrm{~W}-\mathrm{F} 814 \mathrm{~W}$ than the reddest parts of the spheroids.

The observed color difference between the disk and spheroid components is most simply interpreted as a difference in stellar population. Despite the unknown reddening, we can place upper limits on the age of the disk component. Using the Padova single stellar population models (Girardi 2006), for a 10 Gyr old stellar population with metallicities between $[\mathrm{Fe} / \mathrm{H}]$ of -1.7 and -0.4 , the $\mathrm{F} 606 \mathrm{~W}-\mathrm{F} 814 \mathrm{~W}$ color ranges from 0.73 to 0.91 , with redder colors for more metal-rich populations. The difference in color between a 10 and $1 \mathrm{Gyr}$ population is $\lesssim 0.25$ mag regardless of metallicity, suggesting that disks cannot be composed solely of intermediate-age ( $>1$ Gyr) stars. At ages younger than $1 \mathrm{Gyr}$, however, the color quickly becomes bluer. Thus, the $>0.3$ mag color difference is evidence that the disks have stellar populations younger than 1 Gyr. Put another way, we find that the disk components all must have intrinsic colors bluer than F606W $\mathrm{F} 814 \mathrm{~W}=0.65$, which corresponds to single stellar populations younger than 1 Gyr. Our analysis of the spectrum of NGC 4244's nuclear cluster ( $\S 4.2$ ) confirms that stars with ages $\$ 100 \mathrm{Myr}$ are present. Furthermore, each of the nine nuclear cluster spectra analyzed by Walcher et al. (2006) showed evidence for stellar populations with ages $<100 \mathrm{Myr}$.

The suggested interpretation of the color difference as a difference in stellar population may be complicated by a number of factors. First, even if two separate components exist, both likely contribute to the observed color at most locations. This overlap suggests that the actual spread in age is larger than implied by the color differences. Second, the colors could be affected by the presence of dust structures on the same scale as the clusters or by emission-line contribution. Based on the symmetry of the nuclear clusters (e.g., the disk is roughly the same color on both sides of the nucleus) and the spectral results from $\S 4.2$, we believe that the color difference is unlikely to be due solely to dust or emission lines and instead really does reflect a difference in stellar population.

\subsection{Stellar Populations in NGC 4244}

In the previous section we showed that the color offset between the disk and spheroid components of the nuclear cluster indicates that the disk has a systematically younger stellar population. However, because of the uncertain reddening, the ACS colors alone cannot accurately constrain the ages of the stellar populations. In this section we use our APO spectrum and photometry of the nuclear cluster in NGC 4244 from the Sloan Digital Sky Survey (SDSS) and 2MASS to constrain the cluster's age. Before describing the details of this analysis we first describe the photometry.

The nuclear cluster in NGC 4244 is prominent and stands well above the emission from the galaxy disk. Because of this, it was detected as a point source in both the SDSS and 2MASS. These data compliment the spectroscopic data by giving a large wavelength baseline to constrain the mass, reddening, and ages of the nuclear cluster (de Grijs et al. 2003). In both surveys, the source is essentially unresolved, and photometry is determined from PSF fitting. In the SDSS survey, the Data Release 4 object ID is 58773909806344622 , with PSF magnitudes of 17.81, 16.62, $16.18,15.96$, and 15.73 in the $u^{\prime}, g^{\prime}, r^{\prime}, i^{\prime}$, and $z^{\prime}$ filters, respectively. In 2MASS, the object ID is $12172945+3748264$, with magnitudes of $14.36,13.75$, and 13.55 in the $J, H$, and $K$ bands, respectively. The errors on the SDSS magnitudes are $\sim 0.02 \mathrm{mag}$, while the 2MASS observations have errors of $\sim 0.05 \mathrm{mag}$. The difference in resolution and photometric methods between the two surveys means there may be some relative systematic error in the magnitudes. Therefore, any conclusions based on the photometry alone should be considered with caution. We note that because the central pixels of the nuclear cluster were saturated in the ACS data (taken with gain $=1 e^{-}$), we were unable to derive accurate photometry from the HST images.

\subsubsection{Single-Age Fits}

In principle, the photometry and spectrum can be fit with arbitrary stellar populations, but in practice this leads to far too many degeneracies. To simplify the problem, we started by assuming that the NGC 4244 cluster contains stars of a single age and then determined a best-fitting extinction, mass, and $\chi^{2}$ as a function of age from both the photometric and spectroscopic data separately. For the photometric data, we used the single stellar population models from the Padova group (Girardi et al. 2004) using the Marigo (2001) treatment of the asymptotic giant branch. These models have been produced for both the SDSS and 2MASS photometric systems and assume a Kroupa (2001) initial mass function (IMF) between 0.1 and $100 M_{\odot}$. The extinction was calculated in $A_{v}$ assuming $R_{v}=3.1$ using coefficients from Girardi et al. (2004) and Cardelli et al. (1989) for the SDSS and 2MASS data, respectively. For the spectroscopic fitting, single stellar population spectral templates from Bruzual \& Charlot (2003) were used. These templates assume a Chabrier (2003) IMF from 0.1 to $100 M_{\odot}$. They have $1 \AA$ resolution and were redshifted and interpolated to the wavelengths of our observed spectrum. The reddening was applied to the spectrum using the Cardelli et al. (1989) prescription. We fit the feature-rich blue side of the DIS spectrum extending from 3650 to $5500 \AA$, which contains Balmer, $\mathrm{He} \mathrm{I}, \mathrm{Mg}$, and $\mathrm{Ca} \mathrm{H}$ and $\mathrm{K}$ lines (see Fig. 6). The $\mathrm{S} / \mathrm{N}$ of this portion of the spectrum ranged from 20 to 60 . For both the photometry and spectroscopy, we determined the best-fitting mass and reddening at a given age using the IDL CURVEFIT routine. All fits were done assuming a distance modulus of $28.20(D=4.4 \mathrm{Mpc}$; Paper I) and a metallicity of $[\mathrm{Fe} / \mathrm{H}]=-0.4$, similar to the expected current gas-phase metallicity of NGC 4244 (Garnett 2002). This is the same metallicity derived from composite age fits for a majority of the clusters found by Walcher et al. (2006). Furthermore, Rossa et al. (2006) and Walcher et al. (2006) have shown that the inferred age distribution does not depend strongly on the metallicity.

Figure 7 shows the results of our fits for single stellar populations with ages between $10^{7}$ and $10^{10} \mathrm{yr}$ for both the spectroscopy and photometry. The top panel indicates the $\chi^{2}$ of the best-fitting model at each age. The spectrum is best fit by populations younger than $\lesssim 10^{9} \mathrm{yr}$, stellar masses of $(2-3) \times 10^{6} M_{\odot}$, and decreasing extinction with increasing age. The $\chi^{2}$ has a minimum at ages of $\sim(0.8-1.0) \times 10^{9} \mathrm{yr}$ (just before the onset of the red giant branch) and then increases rapidly toward older ages. This results from the template spectrum becoming redder than the observed spectrum. A shallower $\chi^{2}$ minima is also seen in the spectra at 50-100 Myr.

The fits to the photometric data have two comparable $\chi^{2}$ minima at $\sim 10^{8}$ and $\sim 10^{9} \mathrm{yr}$, with the younger age being somewhat preferred. The minima at these two ages is more pronounced in the photometric data than in the spectroscopic data, perhaps as a result of the photometric data's longer wavelength coverage. Combining the photometric and spectroscopic results, the minima in the joint $\chi^{2}$ are at $70 \mathrm{Myr}$ and $0.8 \mathrm{Gyr}$. The masses from the photometric data at these minima are $(3-5) \times 10^{6} M_{\odot}$, somewhat more massive than the spectral data. This difference may result from light lost outside the 1."5 wide slit, uncertainty in the 


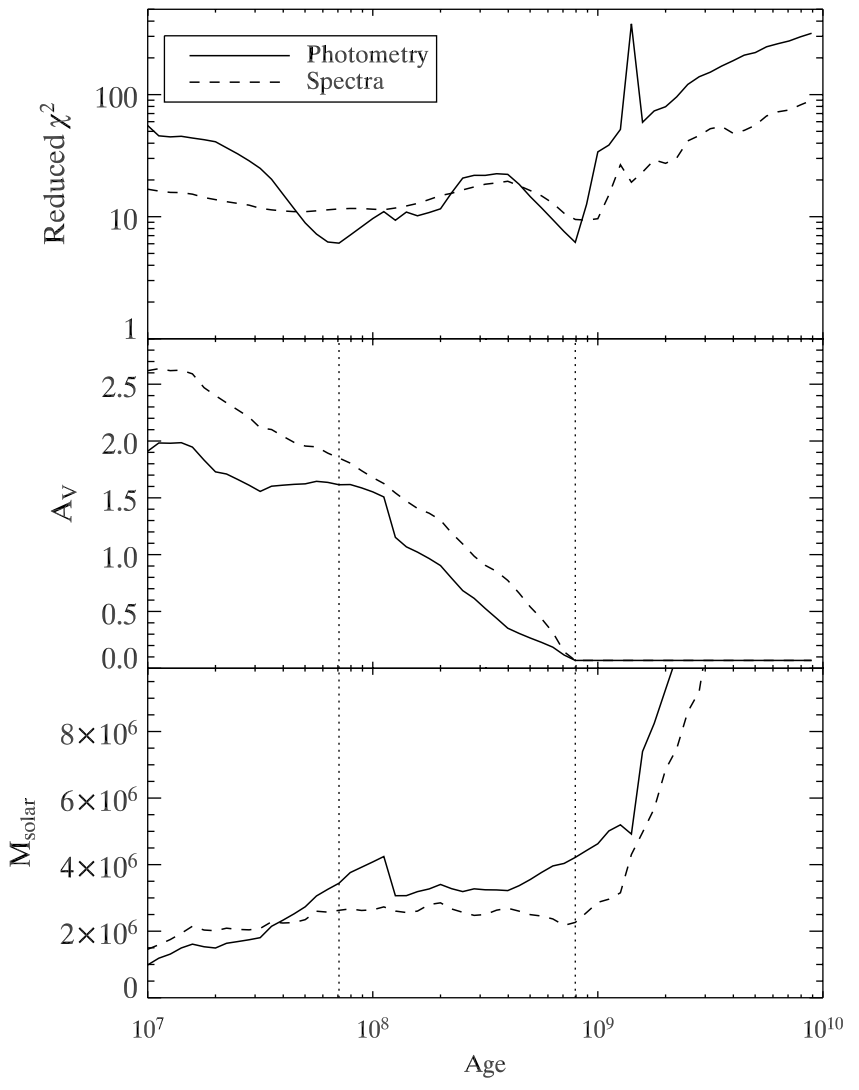

FIG. 7.-Photometric and spectroscopic fits of the mass and reddening of the NGC 4244 nuclear cluster using single stellar population models. Top: Reduced $\chi^{2}$ values of the best-fitting mass and reddening at each age. Middle: Best-fitting reddening. Bottom: Best-fitting mass. The vertical lines indicate the two minima in the joint $\chi^{2}$ distribution at 70 Myr and $0.8 \mathrm{Gyr}$.

absolute calibration of the spectroscopic data, and/or differences in the assumed IMF.

Spectral fits from the two joint $\chi^{2}$ minima are shown in Figure 6 . The younger age appears to fit the bluer part of the spectrum better, while the older age more accurately fits many of the lines and the redder continuum. The $\mathrm{Ca} \mathrm{H}$ line is particularly agesensitive, being much too shallow in the younger age and too deep in the older age.

\subsubsection{Multiage Fits}

The color difference between the disk and spheroid components discussed in $\S 4.1$ and the recent results of Rossa et al. (2006) and Walcher et al. (2006) strongly suggest that nuclear clusters contain stellar populations of multiple ages rather than the single-age populations assumed above. We have tried to constrain the ages and masses of the multiple stellar populations in NGC 4244's nuclear cluster in two ways: (1) by fitting a weighted average of two or three stellar populations of different ages to the spectral and photometric data, and (2) by fitting the spectrum to a constant star formation rate (SFR) model from Bruzual \& Charlot (2003). These approaches both give significantly better fits to the data than single stellar population models, reducing $\chi^{2}$ values by $50 \%$ or more. However, it is also clear that there is no unique solution; the data can be fit equally well by many different combinations of stellar populations and reddening values. Note that we assumed a single extinction $\left(A_{V}\right)$ value for all components.

Of the nine nuclear cluster spectra presented in Walcher et al. (2006), our spectrum of the NGC 4244 nuclear cluster most closely resembles their spectrum of the NGC 7793 nuclear cluster. From their fits to composite stellar populations, they find that the NGC 7793 cluster has an average stellar age of 2 Gyr but has young stars $(\lesssim 100 \mathrm{Myr})$ that make up $10 \%$ of the mass. This is qualitatively similar to what we find below for the NGC 4244 nuclear cluster.

Two-age models. - The simplest stellar population model consistent with the color maps is a two-age stellar population: a young population associated with the disk and an older population associated with the spheroid. Taking the two minima seen in the single stellar population fits at ages of $70 \mathrm{Myr}$ and $0.8 \mathrm{Gyr}$ as a starting point, we explored what combination of two populations gives the best fit to both the spectroscopic and photometric data. For a young component with ages between 50 and $100 \mathrm{Myr}$ and an intermediate-age component with ages between 0.6 and $1.0 \mathrm{Gyr}$, the best fits to the spectroscopy have $A_{V} \sim 0.5$, masses of $(0.5-$ 3) $\times 10^{5} M_{\odot}$ for the young stellar population, total masses of $(2-$ 4) $\times 10^{6} M_{\odot}$, and reduced $\chi^{2}$ values of $\sim 6$. However, for the photometry, the best fits favor an $A_{V}>1$ and masses dominated by the young component. These photometric fits clearly do not reproduce the shape or lines in the spectra; thus, we restrict ourselves to lower $A_{V}$ solutions. When $A_{V}$ is restricted to be less than 1 , the photometric fits are in relatively good agreement with the spectroscopic fits. However, the implied stellar masses for both the young and intermediate-age components are up to 2 times larger than the spectroscopic best-fit models, similar to what was seen in the single-age fits. Overall, we find that ages of 0.1 and 1.0 Gyr do the best job of matching both sets of data. The spectroscopic best fit at these ages is shown in Figure 6 and has $A_{V}=0.46,1.9 \times 10^{5} M_{\odot}$ of 0.1 Gyr old stars, and $3.1 \times 10^{6} M_{\odot}$ of 1 Gyr old stars.

Based on the color map of the nuclear cluster, we expect the younger stellar component in the two-age fits to be associated with the disk. We can test the consistency of this idea by comparing our spectral fits to the luminosities of the disk component decomposed from the $H S T$ data in $\S 3$. Assuming that the disk is dominated by $\sim 70$ Myr old stars, the expected F606W - F814W color from the Padova models is 0.25 (Girardi 2006). The observed disk component color is 0.4 , requiring an extinction $A_{V}=$ $0.47 \mathrm{mag}$. Using this extinction and mass-to-light ratios from the Padova models, we derive a mass for the disk component of $1.2 \times 10^{5} M_{\odot}$. Both the mass and extinction derived from the luminosities of the disk component in the HST data agree with the young stellar mass and extinction determined in the two-age spectral fits. This agreement strongly suggests a link between the blue disk component and the young stellar component that appears to be required to fit the blue spectral features.

Three-age models. - We also explored the sensitivity of our fits to the inclusion of a third old ( $10 \mathrm{Gyr})$ stellar population. We find that a large mass of old stars is consistent with both the photometric and spectroscopic data. Best fits to both data sets have low $A_{V}(<0.4)$; total masses of $\sim 10^{7} M_{\odot}$, with $90 \%$ of that mass in the old component; and $\sim 10^{5} M_{\odot}$ in the young component, similar to the two-age fits. The inclusion of the older component significantly improves the photometric fit to the data (reduced $\chi^{2}=1.7$ ), suggesting older stars may indeed be present. However, the spectral fit is insensitive to this old component; the bestfit spectrum is nearly identical to the two-age fits, despite $90 \%$ of the mass being contained in the old component.

The effects of disk contamination on the photometry and spectra should be minimal given the prominence of the NGC 4244 nuclear cluster. Futhermore, by comparing HST Space Telescope Imaging Spectrograph and ground-based spectra, Walcher et al. 
(2006) show that the stellar population immediately surrounding the nuclear cluster in late-type galaxies seems to have a population similar to the cluster itself. Both Rossa et al. (2006) and Walcher et al. (2006) find some evidence for an underlying old stellar component in many of their nuclear cluster spectra. We note that all the masses quoted here refer to the initial mass in stars required to fit the current spectral data. Due to stellar evolution, the current mass of a cluster dominated by old stars would be $\sim 2$ times lower than the initial mass, given reasonable IMFs.

Constant SFR model.-Using the Bruzual \& Charlot (2003) codes, we generated a model spectrum assuming a constant SFR from 12 Gyr to the present. The best fit of this model to the data gives a current mass of the cluster of $6.2 \times 10^{6} M_{\odot}$ and a reddening of $A_{V}=0.72$. The reduced $\chi^{2}$ of the fit is 5.7 , within $10 \%$ of the reduced $\chi^{2}$ of the best fits obtained for the two- and threeage models. The spectral shape of the constant SFR model appears to be somewhat redder than the data, suggesting the need for either relatively more young stars than in the constant SFR model or a more realistic metallicity evolution, in which the older stars are bluer and more metal-poor. The former point is supported by Walcher et al. (2006), who have shown that in their sample of clusters, the SFR in the past $100 \mathrm{Myr}$ is an order of magnitude greater than the SFR between 0.3 and 20 Gyr. However, this enhancement of recent star formation may result from a bias in their sample toward brighter nuclear clusters.

\subsubsection{Summary of Stellar Populations in the NGC 4244 Nuclear Cluster}

We draw the following conclusions from the spectral and photometric fits of the NGC 4244 nuclear cluster:

1. Multiple stellar populations definitely provide improved fits over single stellar populations for both the spectroscopy and photometry of the NGC 4244 nuclear cluster. However, many different combinations of masses and ages fit the data well.

2. The best-fitting single stellar populations have ages of either $\sim 70$ Myr or $\sim 0.8$ Gyr.

3. The total current mass of the cluster appears to be between 0.2 and $1 \times 10^{7} M_{\odot}$.

4. If we assume that only two ages are present, the youngest ( $\lesssim 100 \mathrm{Myr}$ ) stellar component appears to have $\sim 10^{5} M_{\odot}$ of stars. This mass is consistent with the luminosity of the disk component decomposed using the morphological fits in $\S 3$.

5. For all multiage fits, the mass of stars younger than $100 \mathrm{Myr}$ is a small fraction $(<5 \%)$ of the total mass of the cluster.

6. Stars older than 1 Gyr may be present in large numbers $\left(\sim 10^{7} M_{\odot}\right)$. Inclusion of an old stellar population particularly improves our fits to the SDSS and 2MASS photometric data.

7. A constant SFR fits the data as well as assuming two or three discrete ages.

\section{A DYNAMICAL MASS ESTIMATE FOR THE NGC 4244 CLUSTER}

Emission lines, including $\mathrm{H} \alpha$, [N $\mathrm{NI}]$, [S II], and [O II], are seen in our spectrum of the NGC 4244 nuclear cluster. For the $\mathrm{H} \alpha$ line, the emission component is redshifted relative to the underlying stellar absorption lines (see Fig. 8). We use this offset between the emission and absorption components to obtain a dynamical mass estimate for the NGC 4244 cluster.

Examination of the color map for NGC 4244 in Figure 5 shows a very blue compact source located along the nuclear cluster plane on the left (northeast) side, 0 "'88 (18.6 pc projected) from the nuclear cluster center. This compact blue object has an
F606W - F814W color of $\sim 0.3$, which is $\sim 0.4-0.6$ mag bluer than the disk and spheroid components of the cluster. Assuming moderate reddening and blending with the outer isophotes of the cluster, the color of the blue knot is consistent with this object being an $\mathrm{H}$ II region. $\mathrm{H}$ II regions in our filter combination appear very blue because there are strong emission lines located in the F606W filter and not in the F814W filter. Measurements of $\mathrm{H}$ II regions in NGC 55 (the nearest galaxy in our sample) suggests $\mathrm{H}$ II regions have typical colors of $\mathrm{F} 606 \mathrm{~W}-\mathrm{F} 814 \mathrm{~W}=-0.2$ with an $\sim 0.2$ mag scatter.

This likely $\mathrm{H}$ II region falls within the slit used for our nuclear cluster spectrum. Therefore, we identified this object as the likely source of the emission lines in our spectra. We verified this by extracting spectra from the left (northeast) and right (southwest) sides of the cluster. The left-side spectrum shows significantly enhanced emission lines relative to the right side. However, because the seeing was $\sim 1^{\prime \prime}$, we were unable to spatially separate the emission component from the nuclear cluster. We now proceed under the assumption that the emission lines in our spectrum are associated with this compact blue object.

To determine the relative velocity of the emission and absorption components, we fit the [S II] doublet and [N $\mathrm{NI}]$ line to determine the emission-line velocity and fit the $\mathrm{Na}$ I doublet to determine the absorption-line velocity. We obtained a weighted mean velocity of $270.5 \pm 5.4 \mathrm{~km} \mathrm{~s}^{-1}$ for the emission lines and $246.3 \pm 4.3 \mathrm{~km} \mathrm{~s}^{-1}$ for the $\mathrm{Na}$ I absorption line, assuming air wavelengths for the lines from the Atomic Line List. ${ }^{4}$ This absorption velocity matches the systemic velocity of NGC 4244 (244.4 $\pm 0.3 \mathrm{~km} \mathrm{~s}^{-1}$; Olling 1996) to within the error bars, as would be expected for the nuclear cluster. We note that this H II region is counterrotating with respect to the galaxy disk.

Figure 8 shows the fitted emission and absorption lines, as well as the best fit of absorption + emission for the $\mathrm{H} \alpha$ line after fixing the velocities of the absorption and emission to the values derived from the $\mathrm{Na}$ I, [S II], and [ $\left.\mathrm{N}_{\mathrm{II}}\right]$ lines. This $\mathrm{H} \alpha$ line fit does a good job of replicating the observed line shape, thereby suggesting that the velocities we have derived are correct.

Assuming that the projected radius of the $\mathrm{H}$ in region is close to the true radius, and that it is bound to the NGC 4244 nuclear cluster, the $\Delta v=24.1 \pm 7.0 \mathrm{~km} \mathrm{~s}^{-1}$ offset between emission and absorption implies a nuclear cluster mass of $2.5_{-1.2}^{+1.7} \times 10^{6} M_{\odot}$ within a radius of $19 \mathrm{pc}$. If the object is indeed rotating around the cluster but is in front of or behind the cluster (thus making its projected distance larger), then the mass we derived is a lower limit on the mass of the cluster.

This rough estimate of the dynamical mass of NGC 4244's nuclear cluster is consistent with the stellar masses determined for the one- and two-age fits in $\S 4.2$. It is also consistent with the constant SFR or three-age model fits $\left(M \sim 6 \times 10^{6} M_{\odot}\right)$ if the $\mathrm{H}$ II region is seen in projection and lies $\sim 25 \mathrm{pc}$ from the center of the nuclear cluster.

The estimated mass is also similar to the typical mass determined by Walcher et al. (2005) for late-type galaxy nuclei. They derived masses for nine nuclear clusters from the Böker et al. (2002) sample by combining size and velocity dispersion measurements. Their total range of masses is $8 \times 10^{5}$ to $6 \times 10^{7} \mathrm{M}_{\odot}$, with seven of the nine clusters having masses between 0.1 and $1 \times 10^{7} M_{\odot}$. We note that they have determined these masses assuming spherical symmetry; if these clusters have disk components similar to those we have observed here, the masses derived by Walcher et al. (2005) may be underestimates, since they do not take into account the rotational support that would be expected in

\footnotetext{
${ }^{4}$ Available at http://www.pa.uky.edu/ peter/atomic/.
} 

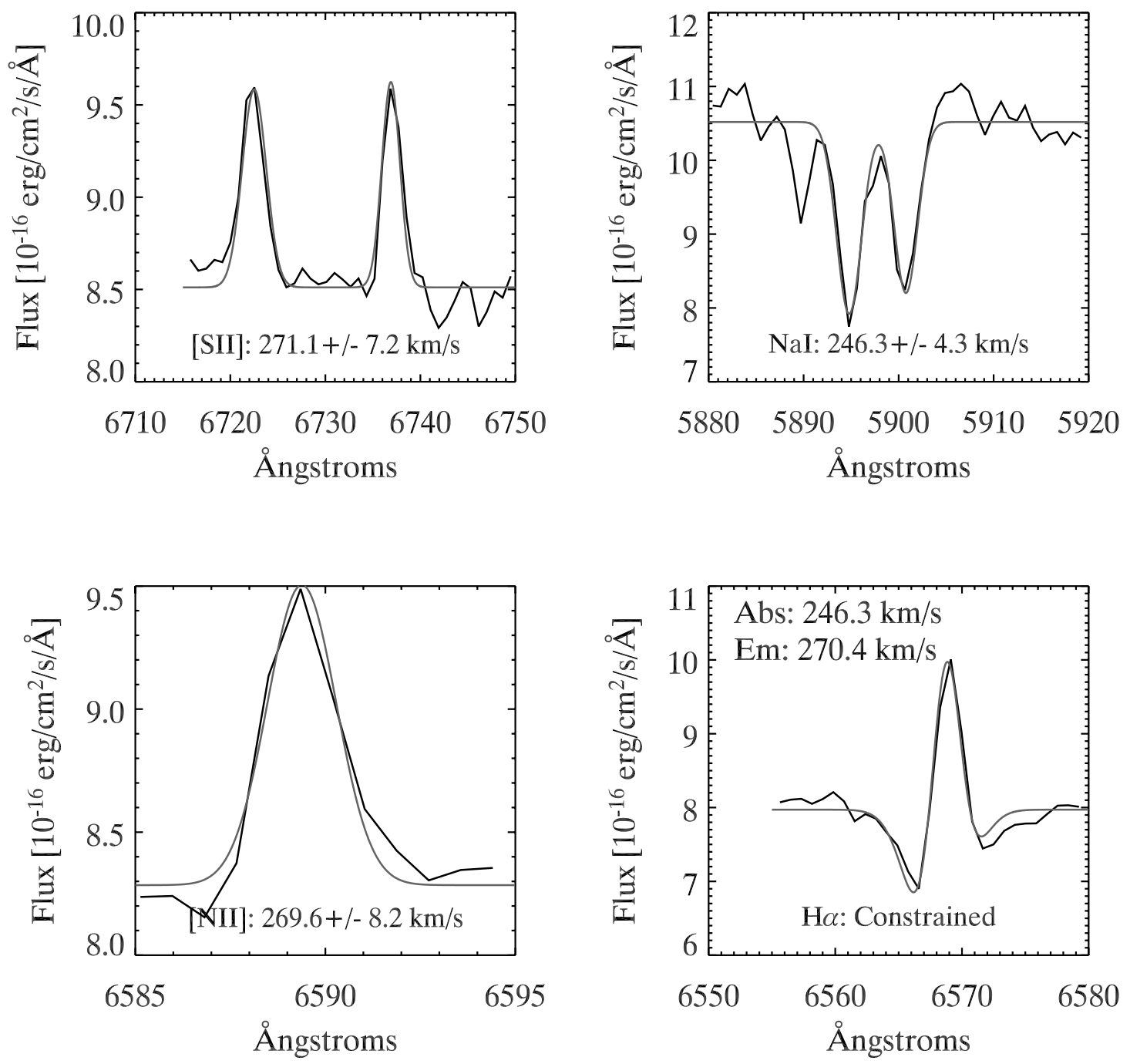

Fig. 8. - Spectral lines used to derive the relative offset between emission and absorption. Dark lines show the actual spectrum, while light lines show the bestfitting Gaussian or double Gaussian model. The number in each panel gives the fitted velocity and error bar. The spectra has a $\mathrm{S} / \mathrm{N} \sim 35$ pixel ${ }^{-1}$.

a disk. Given that their clusters contain young stellar populations (Walcher et al. 2006), this effect could be significant.

\section{NGC 4206: POSSIBLE INDICATION OF AN AGN COMPONENT}

In this section we present evidence that the nuclear cluster in NGC 4206 may contain an AGN component. This evidence is very tentative and requires confirmation with follow-up observations.

An SDSS spectrum (MJD 53,149, plate 1612, fiber 603) exists for the central regions of NGC 4206. Due to the $3^{\prime \prime}$ diameter $(\sim 250 \mathrm{pc})$ aperture, this spectrum is dominated by the light from the central regions of the galaxy and not by the nuclear cluster. In addition to a dominant old stellar spectrum, narrow $\mathrm{H} \alpha$, [N II], and [S II] emission are clearly seen. No broad emission lines are seen. Examining the HST color map, the center of the nuclear cluster is the bluest area anywhere within the SDSS spectral aperture. Since emission-line sources are very blue in our filter combination, the central portion of the nuclear cluster is the likely source of the emission lines. The emission lines are not offset in velocity from the absorption spectrum (as was seen in NGC 4244), further supporting their nuclear origin.

The ionizing source of the observed emission lines could be either star formation or AGN activity at the center of the nuclear cluster. These two ionizing sources can be distinguished using the line ratio of [N $\mathrm{II}]$ and $\mathrm{H} \alpha$. Because the $\mathrm{H} \alpha$ line is contaminated by stellar absorption, we model the underlying stellar continuum with a single stellar population $\sim 3 \mathrm{Gyr}$ in age with $A_{V} \sim 0.5$. After subtracting this spectrum, the line ratio of the remaining emission lines is $\log ([\mathrm{N} \mathrm{II}] / \mathrm{H} \alpha) \sim-0.3$. Based on the recent analysis of SDSS spectra by Obric et al. (2006), this line ratio suggests that the source is a "mixed" starburst/AGN. NGC 4206 may therefore host a small AGN within the core of the nuclear star cluster. If confirmed by follow-up observations, this would make it only the second late-type spiral known to have both a SMBH and a massive nuclear star cluster, the other being NGC 4395, an Sd galaxy with $M_{B} \sim-17.5$ hosting a nuclear cluster with $M_{I} \sim-10.1$ (Matthews et al. 1999) and a black hole with a mass of $\sim 10^{4}-10^{5} M_{\odot}$ (Filippenko \& Ho 2003). In earlier type spirals, other examples exist of the coexistence of nuclear star clusters and AGNs (e.g., Thatte et al. 1997; Davies et al. 2006). We plan to test for the presence of AGN components in all of our multicomponent nuclear cluster candidates with near-IR integral field unit spectroscopy using adaptive optics.

\section{DISCUSSION}

We now discuss how our data constrain the formation mechanisms of nuclear clusters. We suggest a formation mechanism in which stars in nuclear clusters form episodically in disks and then, 
over time, lose angular momentum or are heated vertically, ending up in a more spheroidal distribution. After discussing this mechanism in some detail, we examine the relation of nuclear clusters to other massive star clusters and SMBHs.

\subsection{Formation Mechanisms}

Our observations of multicomponent clusters place significant constraints on nuclear cluster formation mechanisms. The presence of young nuclear cluster disks $(<1 \mathrm{Gyr})$ aligned with the disks of the host galaxies strongly suggests that they are formed in situ from gas accreted into the nuclear regions. We have shown in $\S 3.2$ that these disks may be a common feature of nuclear clusters. Such disks cannot be formed via the accretion of globular clusters by dynamical friction. While globular cluster accretion very likely occurs, this mechanism has been shown to be relatively inefficient in late-type galaxies (Milosavljević 2004). We propose here a scenario in which the dominant formation mechanism for nuclear clusters is via in situ formation in disks. Although the mechanism will need to be tested with detailed simulations, it is consistent not just with our data but with both the evidence for young and composite stellar populations in many nuclear clusters (e.g., Walcher et al. 2006) and the direct detection of a molecular gas disk coincident with the nuclear cluster in IC 342 (Schinnerer et al. 2003).

On a large scale $(\sim 1 \mathrm{kpc})$, both dissipative merging of molecular clouds and magnetorotational instabilities have been shown to be capable of supplying the nucleus of a late-type galaxy with sufficient gas to form nuclear clusters (Milosavljević 2004; Bekki et al. 2006). Our proposed in situ formation mechanism is concerned with what occurs once the gas reaches the center. Our model is also fully consistent with the suggestion by Walcher et al. (2006) that an initial seed star cluster is required for subsequent nuclear star cluster formation.

The details of an in situ formation mechanism can be constrained by our observations. To explain the presence of redder/ older spheroids in the multicomponent clusters and the absence of disk components in a majority of our nuclear clusters, we propose a model in which nuclear cluster disks form episodically and are transformed into a rounder distribution over time due to loss of angular momentum or dynamical heating. There are two important timescales in this model: (1) the period of time between star formation episodes and (2) the time it takes stars in a disk morphology to end up in a spheroidal distribution. We examine these two timescales in detail below.

\subsubsection{Period between Star Formation Episodes}

A number of authors have previously suggested that star formation in the nuclear regions of late-type spirals may be episodic. The physical motivation for episodic star formation is that a massive star formation event will feedback on the local environment, preventing star formation for the next $\sim 10^{8} \mathrm{yr}$ (Milosavljevic 2004). Although this feedback may destroy the gas disk and shut off star formation, it does not disrupt the disk of recently formed stars, whose lifetime we discuss in $\S$ 7.1.2.

Assuming that the present epoch is not a special time in the life of the nuclear clusters, one can estimate the period on which star formation recurs. Observationally, Davidge \& Courteau (2002) argue for a period of $\sim 0.3 \mathrm{Gyr}$ based on the detection frequency of $\mathrm{Pa} \alpha$ emission in galaxy nuclei. Schinnerer et al. (2003) also determine a recurrence period of a few hundred Myr to $1 \mathrm{Gyr}$ based on the gas accretion rates observed in IC 342's nucleus. More recently, from analysis of the youngest stellar components in the spectrum, Walcher et al. (2006) have found the typical time between star formation bursts to be $\sim 70$ Myr with a typical mass of roughly $1.6 \times 10^{5} M_{\odot}$.

Our observations of the NGC 4244 nuclear cluster are consistent with these previous estimates. Based on the $\sim 10^{5} M_{\odot}$ of stars in the young component ( $\S 4.2$ ), approximately 25 episodes of disk formation must have occurred to build up the $2.5 \times 10^{6} M_{\odot}$ of the cluster $(\S 5)$. If this occurs over a Hubble time, then the period between bursts is $\sim 0.5 \mathrm{Gyr}$.

\subsubsection{Timescale of Disk Transformation}

Given that nuclear cluster disks are observed in $<50 \%$ of nuclear clusters, and that these disks all have stellar populations younger than $1 \mathrm{Gyr}$, a mechanism must exist that destroys or disrupts this disk on a timescale shorter than $\sim 1$ Gyr. We suggest that angular momentum loss or dynamical heating causes the disks to be transformed over time, thus creating the more spheroidal, older components seen in our nuclear clusters. Although mechanisms of angular momentum loss have not been studied previously in nuclear clusters, these objects are dynamically similar to globular clusters, for which the theory of rotation and rotational evolution has been well-developed (Agekian 1958; King 1961; Shapiro \& Marchant 1976; Fall \& Frenk 1985; Davoust \& Prugniel 1990; Longaretti \& Lagoute 1997).

As they age, star clusters flattened due to rotation will become increasingly round due to the preferential evaporation of high angular momentum stars (Agekian 1958). We can compare the timescale of this angular momentum loss to the expected lifetimes of our disks. The timescale over which the ellipticity of a rotating stellar cluster decreases is $\gtrsim 40$ times the median relaxation time, $\tau_{r h}$ (Shapiro \& Marchant 1976; Fall \& Frenk 1985). For globular clusters in the Milky Way, $\tau_{r h}$ is typically $\sim 10^{8}$ yr. However, this timescale is longer for more massive clusters like $\omega$ Cen, with $\tau_{r h}=3.5$ Gyr (Shapiro \& Marchant 1976). For the NGC 4244 nuclear cluster we have calculated a median relaxation time for the whole cluster of 2 Gyr (eq. [8-72] of Binney \& Tremaine 1987). For just the disk component, assuming that it has a mass of $\sim 10^{5} M_{\odot}$ and a half-mass radius of $\sim 3 \mathrm{pc}$, we get $\tau_{r h}=4 \times 10^{8} \mathrm{yr}$. These relaxation times suggest that the timescale for evaporation of angular momentum in NGC 4244 is in the range of 16-80 Gyr. This timescale is significantly longer than the hundreds of Myr over which we expect the disk to be transformed into a spheroidal component.

It therefore appears that angular momentum loss due to processes internal to the clusters is unlikely to transform the disk components into spheroids on sufficiently short timescales. Instead, we must look to external processes that can affect the dynamical evolution of the nuclear cluster. We have identified a few alternative mechanisms for transforming the stellar disk component into a spheroid. These include (1) small angular offsets of the nuclear disk from the plane of the galaxy, (2) slight positional offsets of the cluster from the center of the galaxy potential, and (3) torques on the stellar disk from successive gas accretion. A forthcoming paper will discuss this problem in more detail.

\subsubsection{Rings}

We have been assuming thus far that stars are formed initially in disks, as suggested by our observations of NGC 4244 and NGC 4206. However, in $\S 3.1 .3$, we show that one of the three multicomponent nuclear clusters, in IC 5052, is best fit by a spheroid + ring model with inner and outer ring radii of 6.8 and 11.8 pc. We discuss here how such a ring could form and examine rings in the context of our proposed nuclear cluster formation model. 
Based on an elongated near-infrared component in IC 342, Böker et al. (1997) suggested that the observed molecular ring (with a radius ${ }^{5}$ of $\sim 65 \mathrm{pc}$ ) results from an inner Lindblad resonance with a stellar bar. If a similar mechanism is at work in forming the ring in IC 5052, it would require the star cluster itself to have a barred disk morphology. From HST imaging of nuclear clusters in face-on spirals, there is no evidence for bars on these scales (Böker et al. 2004). A ring could also result from tidal stripping of an infalling molecular cloud or star cluster. Assuming a preexisting cluster with mass of $3 \times 10^{6} M_{\odot}$ and a $10^{5} M_{\odot}$ molecular cloud, the molecular cloud would be tidally stripped between radii of 8 and 20 pc for molecular cloud number densities ranging between $5 \times 10^{4}$ and $5 \times 10^{3} \mathrm{~cm}^{-3}$. Thus, tidal stripping of a molecular cloud is a feasible mechanism for creating a ring such as that in IC 5052.

Episodic star formation occurring at least occasionally in rings is consistent with the proposed formation model. The ring in IC 5052 is internal to the cluster itself, so after loss of angular momentum or dynamical heating, its stars would still contribute to the stellar distribution of the spheroid. The radius of the ring depends on the current mass of the star cluster in both mechanisms for ring formation as described above. This correlation might provide a natural explanation for the clear correlation of nuclear cluster size and luminosity observed in dwarf elliptical galaxies by Côté et al. (2006). A similar explanation could work for formation in disks if the size of the disks depends on the mass of the nuclear cluster. Detailed modeling of disk/ring formation and dissipation would be required to test the feasibility of this idea.

\subsubsection{Other Formation Scenarios}

Our proposed scenario naturally explains the formation of multicomponent clusters. However, other scenarios may also be consistent with our observations. While our observations seem to require some in situ formation to explain the presence of young stellar disks, they do not rule out the possibility that the multicomponent nuclear clusters were formed in part from the merging of globular clusters (e.g., Tremaine et al. 1975; Lotz et al. 2001). In this "hybrid" scenario, the young disk components may be a late-time veneer on an already massive cluster formed from merged globular clusters. However, we note that Milosavljević (2004) has calculated that the timescale for dynamical friction in latetype spirals is $\gtrsim 3 \times 10^{9}$ yr even for relatively massive clusters $\left(10^{6} M_{\odot}\right)$ located within the central kiloparsec of the galaxy. This quantity increases for less massive and more distant clusters. He concludes that this timescale is thus too long to be the dominant mechanism for nuclear cluster formation, and he shows that magnetorotational instabilities can transport sufficient quantities of gas into the galaxy center to account for the observed nuclear clusters in late-type spirals.

\subsection{Relationship to Other Massive Star Clusters}

Our observations have implications beyond just the formation of nuclear star clusters in late-type galaxies. We briefly review here the possible connections between late-type spiral nuclei and dwarf elliptical nuclei and the evidence that some massive globular clusters and UCDs are stripped nuclear clusters.

\subsubsection{The Relation between Spiral and $d E$ Nuclei}

The recent study by Côté et al. (2006) shows that the nuclei of $\mathrm{dE}$ and late-type spiral galaxies have comparable sizes, lumi-

\footnotetext{
5 The radius of their ring was recalculated assuming the Cepheid distance to IC 342 of 3.3 Mpc derived by Saha et al. (2002).
}

nosities, and frequencies of detection (see also Fig. 3). They argue that this similarity suggests a nuclear cluster formation mechanism that does not depend strongly on galaxy properties. However, the formation scenario suggested by our observations for nuclear clusters in late-type galaxies cannot take place in the gasfree $\mathrm{dE}$ galaxies. We therefore interpret the similarity of the nuclei in these galaxies as support for $\mathrm{dE}$ galaxies evolving from lowmass spiral and irregular galaxies (e.g., Davies \& Phillipps 1988). In this scenario, a dE, $\mathrm{N}$ galaxy would result from a nucleated latetype spiral galaxy after depletion of gas through star formation or galaxy harassment (Moore et al. 1996). If so, then the ages of the nuclear clusters in $\mathrm{dE}$ galaxies could constrain the epoch when these systems were stripped.

\subsubsection{Massive Star Clusters as Stripped Galaxy Nuclei}

There is direct evidence that some massive globular clusters are associated with stripped galaxies. The Galactic globular cluster M54 appears to be the nucleus of the Sgr dSph (Layden \& Sarajedini 2000), and six of the most massive globular clusters in NGC 5128 appear to be surrounded by extratidal light (Harris et al. 2002; Martini \& Ho 2004).

Stripped nuclear clusters have also been invoked to explain the multiple stellar populations observed in the most massive nearby globular clusters (e.g., Gnedin et al. 2002). These observations include the large metallicity spread (Smith 2004) and double main sequence (Bedin et al. 2004) in $\omega$ Cen and the spread in red giant branch colors for G1 in Andromeda (Meylan et al. 2001). More recently, Harris et al. (2006) have observed a trend toward redder colors with increasing luminosity for the brightest blue globular clusters in massive elliptical galaxies. This trend is interpreted as evidence for self-enrichment in these clusters pointing to an extended star formation history more typical of nuclear clusters than globular clusters. A similar redward trend is seen for dE nuclei by Côté et al. (2006). Self-enrichment is naturally explained in an in situ formation mechanism but is not easily explained if these clusters are formed by merging less massive clusters via dynamical friction.

The UCD galaxies recently found in the Fornax (Phillipps et al. 2001; Drinkwater et al. 2003) and Virgo (Jones et al. 2006) clusters may also be related to nuclear star clusters. One possible mechanism for their formation is "galaxy threshing," in which nucleated galaxies lose their envelopes, leaving behind only their nuclei as UCDs (Bekki et al. 2001). In the $M_{I}$ versus $\log \left(r_{\text {eff }}\right)$ diagram in Figure 3, NGC 4206's nuclear cluster has sufficient size and luminosity, even after modest age fading, to be a UCD progenitor. The galaxy-threshing scenario has also been shown to be feasible by Côté et al. (2006) for dwarf elliptical nuclei, although De Propris et al. (2005) suggest that dE,Ns are in general more compact than UCDs. One of the galaxy nuclei they study does have properties similar to those of the UCDs, as well as possessing spiral arms, which indicate that the galaxy may be an anemic spiral.

\subsection{Connection to Black Holes}

As residents of the same neighborhoods, it is natural to suspect that there may be some connection between nuclear clusters and SMBHs. The presence of SMBHs in the nuclei of $>30$ galaxies has been confirmed through dynamical measurements of their masses (reviewed by Ferrarese \& Ford 2005). The masses of SMBHs are known to correlate with the mass and/or velocity dispersion of the bulge component (the $M_{\bullet}-\sigma$ relation; Ferrarese $\&$ Merritt 2000; Gebhardt et al. 2000). Recent work on nuclear clusters in both spiral and elliptical galaxies has shown a remarkably similar trend (Rossa et al. 2006; Côté et al. 2006; Ferrarese 
et al. 2006; Wehner \& Harris 2006). For a large sample of nucleated elliptical galaxies, Côté et al. (2006) have shown that the nucleus accounts for $0.30 \% \pm 0.04 \%$ of the total galaxy mass. This fraction is very similar to the fraction of galaxy mass found in the SMBHs (Ferrarese et al. 2006). For this reason, Côté et al. (2006), Ferrarese et al. (2006), and Wehner \& Harris (2006) suggest that nuclear clusters and SMBHs be thought of as a single class of central massive objects. They suggest a transition at $\sim 10^{7} M_{\odot}$ between nuclear clusters and SMBHs. However, the detection of numerous lower mass SMBHs at the centers of earlyand late-type dwarf galaxies (Barth et al. 2005; Greene et al. 2005), and the coexistence of nuclear star clusters and SMBHs in NGC 4395 (Filippenko \& Ho 2003) and perhaps in NGC 4206 (§ 6), suggest that this transition may not be abrupt.

The formation of black holes in the centers of nuclear clusters may be related to the IMBHs found in the centers of massive nonnuclear star clusters. A $1.7 \times 10^{4} M_{\odot}$ black hole has been reported by Gebhardt et al. (2005) in the globular cluster G1 in Andromeda (which is also a possible stripped nuclei), based on stellar dynamics. A $10^{2}-10^{4} M_{\odot}$ black hole has also been identified in one of M82's young massive clusters, based on X-ray observations (Patruno et al. 2006). These black holes are thought to form either by early merging of massive young stars within the cluster or by dynamical interaction in the cores of the clusters over long time periods (Miller \& Colbert 2004 and references therein). If IMBH formation is common in dense massive clusters, then nuclear clusters should frequently host black holes as well. Subsequent mergers of many IMBHs could then form SMBHs (Miller \& Colbert 2004). As the mass of the SMBH increases, these mergers will likely destroy the nuclear clusters (see $\S 5.2 .2$ of Côté et al. 2006), distributing their stars into the bulge.

\section{SUMMARY}

We have examined the nuclear regions of 14 nearby galaxies using HST ACS images in the F606W and F814W filters. We find nuclear cluster candidates within $2^{\prime \prime}$ of the photocenter of nine of these galaxies. We have also obtained multiband photometry and a spectrum of the NGC 4244 nuclear cluster, the nearest object in our sample. Analyzing these nuclear clusters, we find the following:

1. From the edge-on perspective, three of the nine cluster candidates (IC 5052, NGC 4206, and NGC 4244) have morphologies suggesting that they have both disk and spheroid components. These components are very compact, with scale lengths and half-light radii of 2-30 pc. Star clusters with multiple visible components have not previously been seen.

2. The three multicomponent nuclear clusters have elongations oriented to within $\sim 10^{\circ}$ of the major axis of the galaxy.
3. The magnitudes and sizes of the clusters in our edge-on sample are very similar to those observed in other face-on, latetype galaxies (Böker et al. 2004) and dE galaxies (Côté et al. 2006).

4. The spheroids of the multicomponent nuclear clusters are $0.3-0.6$ mag redder than the disks in F606W - F814W. This color difference implies that the disks are populated with stars $<1$ Gyr in age.

5. Fitting single stellar population models to the spectral and photometric data of the NGC 4244 nuclear cluster, we find that it is best fit by ages of $\sim 70 \mathrm{Myr}$ or $\sim 0.8 \mathrm{Gyr}$. The implied total mass of the cluster is $\sim 3 \times 10^{6} M_{\odot}$.

6. A combination of two or more stellar populations significantly improves the fit to the NGC 4244 nuclear cluster spectral and photometric data. In the multiage fits, the youngest ( $\leqslant 100 \mathrm{Myr}$ ) stellar population matches the luminosity of the disk component and appears to make up $\lesssim 5 \%$ of the total mass of the cluster. As much as $\sim 10^{7} M_{\odot}$ of old (10 Gyr) stars may also be present in the cluster. A constant star formation rate over the last $12 \mathrm{Gyr}$ also provides a surprisingly good fit.

7. Using an $\mathrm{H}$ II region $19 \mathrm{pc}$ from the center of the NGC 4244 nuclear cluster, we measure the velocity offset of emission and absorption components in our spectrum to obtain a lower limit to the dynamical mass for the nuclear cluster of $2.5_{-1.2}^{+1.7} \times 10^{6} M_{\odot}$.

8. Analysis of emission lines in the NGC 4206 nucleus suggests that it may host an AGN, as well as a stellar cluster.

These observational results strongly support an in situ formation model for nuclear star clusters. We suggest a specific model in which stars are formed episodically when gas accretes into a compact nuclear disk. After formation the stars gradually lose angular momentum and eventually end up in a spheroidal component. We show that a duty cycle for star formation of $\sim 0.5 \mathrm{Gyr}$ is consistent with our and previous observations. The angular momentum must also be lost on this timescale, which we suggest may occur due to interactions of the nuclear cluster with the surrounding galaxy potential.

The authors would like to thank the anonymous referee, CarlJakob Walcher, Thomas Puzia, Tom Quinn, and Nate Bastian for their helpful comments in improving this manuscript. We also thank Leo Girardi for his help in interpreting models, Andrew West for his help with SDSS, Kevin Covey for fruitful discussions, and Roelof de Jong for his ongoing support. This work has been supported by HST-AR-10309, HST-GO-9765, the Sloan Foundation, and NSF CAREER grant AST 02-38683.
Agekian, T. A. 1958, Soviet Astron., 2, 22

Barth, A. J., Greene, J. E., \& Ho, L. C. 2005, ApJ, 619, L151

Bastian, N., Saglia, R. P., Goudfrooij, P., Kissler-Patig, M., Maraston, C., Schweizer, F., \& Zoccali, M. 2006, A\&A, 448, 881

Beasley, M. A., Strader, J., Brodie, J. P., Cenarro, A. J., \& Geha, M. 2006, AJ, 131,814

Bedin, L. R., Piotto, G., Anderson, J., Cassisi, S., King, I. R., Momany, Y., \& Carraro, G. 2004, ApJ, 605, L125

Bekki, K., \& Chiba, M. 2004, A\&A, 417, 437

Bekki, K., Couch, W. J., \& Drinkwater, M. J. 2001, ApJ, 552, L105

Bekki, K., Couch, W. J., \& Shioya, Y. 2006, ApJ, 642, L133

Binney, J., \& Tremaine, S. 1987, Galactic Dynamics (Princeton: Princeton Univ. Press)

Böker, T., Forster-Schreiber, N. M., \& Genzel, R. 1997, AJ, 114, 1883

Böker, T., Krabbe, A., \& Storey, J. W. V. 1998, ApJ, 498, L115

\section{REFERENCES}

Böker, T., Laine, S., van der Marel, R. P., Sarzi, M., Rix, H.-W., Ho, L. C., \& Shields, J. C. 2002, AJ, 123, 1389

Böker, T., Sarzi, M., McLaughlin, D. E., van der Marel, R. P., Rix, H.-W., Ho, L. C., \& Shields, J. C. 2004, AJ, 127, 105

Bottinelli, L., Gouguenheim, L., Paturel, G., \& de Vaucouleurs, G. 1985, A\&AS, 59, 43

Bottinelli, L., Gouguenheim, L., \& Teerikorpi, P. 1988, A\&A, 196, 17

Bruzual, G., \& Charlot, S. 2003, MNRAS, 344, 1000

Cardelli, J. A., Clayton, G. C., \& Mathis, J. S. 1989, ApJ, 345, 245

Carlson, M. N., \& Holtzman, J. A. 2001, PASP, 113, 1522

Carollo, C. M., Stiavelli, M., Seigar, M., de Zeeuw, P. T., \& Dejonghe, H. 2002, AJ, 123, 159

Chabrier, G. 2003, PASP, 115, 763

Côté, P., et al. 2006, ApJS, 165, 57

Davidge, T. J., \& Courteau, S. 2002, AJ, 123, 1438 
Davies, J. I., \& Phillipps, S. 1988, MNRAS, 233, 553

Davies, R., et al. 2006, ApJ, 646, 754

Davoust, E., \& Prugniel, P. 1990, A\&A, 230, 67

de Grijs, R., Fritze-v. Alvensleben, U., Anders, P., Gallagher, J. S., Bastian, N., Taylor, V. A., \& Windhorst, R. A. 2003, MNRAS, 342, 259

De Propris, R., Phillipps, S., Drinkwater, M. J., Gregg, M. D., Jones, J. B., Evstigneeva, E., \& Bekki, K. 2005, ApJ, 623, L105

Drinkwater, M. J., Gregg, M. D., Hilker, M., Bekki, K., Couch, W. J., Ferguson, H. C., Jones, J. B., \& Phillipps, S. 2003, Nature, 423, 519

Fall, S. M., \& Frenk, C. S. 1985, in IAU Symp. 113, Dynamics of Star Clusters, ed. J. Goodman \& P. Hut (Dordrecht: Reidel), 285

Ferrarese, L. 2002, ApJ, 578, 90

Ferrarese, L., \& Ford, H. 2005, Space Sci. Rev., 116, 523

Ferrarese, L., \& Merritt, D. 2000, ApJ, 539, L9

Ferrarese, L., et al. 2006, ApJ, 644, L21

Filippenko, A. V., \& Ho, L. C. 2003, ApJ, 588, L13

Garnett, D. R. 2002, ApJ, 581, 1019

Gavazzi, G., Boselli, A., Scodeggio, M., Pierini, D., \& Belsole, E. 1999, MNRAS, 304, 595

Gebhardt, K., Rich, R. M., \& Ho, L. C. 2002, ApJ, 578, L41 . 2005, ApJ, 634, 1093

Gebhardt, K., et al. 2000, ApJ, 539, L13

Girardi, L. 2006, Padova Database of Stellar Evolutionary Tracks and Isochrones (Padua: Oss. Astron. Padova), http://pleiadi.pd.astro.it

Girardi, L., Grebel, E. K., Odenkirchen, M., \& Chiosi, C. 2004, A\&A, 422, 205

Gnedin, O. Y., Zhao, H., Pringle, J. E., Fall, S. M., Livio, M., \& Meylan, G. 2002, ApJ, 568, L23

Grant, N. I., Kuipers, J. A., \& Phillipps, S. 2005, MNRAS, 363, 1019

Greene, J. E., Barth, A. J., \& Ho, L. C. 2005, in QSO Hosts: Evolution and Environment, ed. P. D. Barthel \& D. B. Sanders, submitted (astro-ph/0511810) Harris, W. E. 1996, AJ, 112, 1487

Harris, W. E., Harris, G. L. H., Holland, S. T., \& McLaughlin, D. E. 2002, AJ, 124,1435

Harris, W. E., Whitmore, B. C., Karakla, D., Okoń, W., Baum, W. A., Hanes, D. A., \& Kavelaars, J. J. 2006, ApJ, 636, 90

Ho, L. C., Filippenko, A. V., \& Sargent, W. L. 1995, ApJS, 98, 477

Huxor, A. P., Tanvir, N. R., Irwin, M. J., Ibata, R., Collett, J. L., Ferguson, A. M. N., Bridges, T., \& Lewis, G. F. 2005, MNRAS, 360, 1007

Jensen, J. B., Tonry, J. L., Barris, B. J., Thompson, R. I., Liu, M. C., Rieke, M. J., Ajhar, E. A., \& Blakeslee, J. P. 2003, ApJ, 583, 712

Jones, J. B., et al. 2006, AJ, 131, 312

King, I. 1961, AJ, 66, 68

Kroupa, P. 2001, MNRAS, 322, 231

Larsen, S. S. 1999, A\&AS, 139, 393

Lauer, T. R., Faber, S. M., Ajhar, E. A., Grillmair, C. J., \& Scowen, P. A. 1998, AJ, 116, 2263

Layden, A. C., \& Sarajedini, A. 2000, AJ, 119, 1760

Long, K. S., Charles, P. A., \& Dubus, G. 2002, ApJ, 569, 204

Longaretti, P.-Y., \& Lagoute, C. 1997, A\&A, 319, 839

Lotz, J. M., Miller, B. W., \& Ferguson, H. C. 2004, ApJ, 613, 262
Lotz, J. M., Telford, R., Ferguson, H. C., Miller, B. W., Stiavelli, M., \& Mack, J. 2001, ApJ, 552, 572

Mackey, A. D., \& van den Bergh, S. 2005, MNRAS, 360, 631

Maoz, D., Barth, A. J., Sternberg, A., Filippenko, A. V., Ho, L. C., Macchetto, F. D., Rix, H.-W., \& Schneider, D. P. 1996, AJ, 111, 2248

Marigo, P. 2001, A\&A, 370, 194

Martini, P., \& Ho, L. C. 2004, ApJ, 610, 233

Mastropietro, C., Moore, B., Mayer, L., Debattista, V. P., Piffaretti, R., \& Stadel, J. 2005, MNRAS, 364, 607

Matthews, L. D., et al. 1999, AJ, 118, 208

Meylan, G., \& Mayor, M. 1986, A\&A, 166, 122

Meylan, G., Sarajedini, A., Jablonka, P., Djorgovski, S. G., Bridges, T., \& Rich, R. M. 2001, AJ, 122, 830

Mieske, S., Hilker, M., \& Infante, L. 2002, A\&A, 383, 823

Miller, M. C., \& Colbert, E. J. M. 2004, Int. J. Mod. Phys. D, 13, 1

Milosavljević, M. 2004, ApJ, 605, L13

Moore, B., Katz, N., Lake, G., Dressler, A., \& Oemler, A. 1996, Nature, 379, 613

Obric, M., et al. 2006, MNRAS, 370, 1677

Olling, R. P. 1996, AJ, 112, 457

Patruno, A., Portegies Zwart, S. F., Dewi, J., \& Hopman, C. 2006, MNRAS, 370, L6

Paturel, G., Theureau, G., Bottinelli, L., Gouguenheim, L., Coudreau-Durand, N., Hallet, N., \& Petit, C. 2003, A\&A, 412, 57

Paturel, G., et al. 1995, in Information and On-Line Data in Astronomy, ed. D. Egret \& M. A. Albrecht (Dordrecht: Kluwer), 115

Phillipps, S., Drinkwater, M. J., Gregg, M. D., \& Jones, J. B. 2001, ApJ, 560, 201

Rossa, J., van der Marel, R. P., Boeker, T., Gerssen, J., Ho, L. C., Rix, H.-W., Shields, J. C., \& Walcher, C.-J. 2006, AJ, 132, 1074

Saha, A., Claver, J., \& Hoessel, J. G. 2002, AJ, 124, 839

Sarzi, M., Rix, H.-W., Shields, J. C., Ho, L. C., Barth, A. J., Rudnick, G., Filippenko, A. V., \& Sargent, W. L. W. 2005, ApJ, 628, 169

Schinnerer, E., Böker, T., \& Meier, D. S. 2003, ApJ, 591, L115

Schlegel, D. J., Finkbeiner, D. P., \& Davis, M. 1998, ApJ, 500, 525

Seth, A. C., Dalcanton, J. J., \& de Jong, R. S. 2005, AJ, 129, 1331 (Paper I)

Shapiro, S. L., \& Marchant, A. B. 1976, ApJ, 210, 757

Sirianni, M., et al. 2005, PASP, 117, 1049

Smith, V. V. 2004, in Origin and Evolution of the Elements, ed. A. McWilliam $\&$ M. Rauch (Cambridge: Cambridge Univ. Press), 186

Thatte, N., Quirrenbach, A., Genzel, R., Maiolino, R., \& Tecza, M. 1997, ApJ, 490, 238

Tonry, J. L., Dressler, A., Blakeslee, J. P., Ajhar, E. A., Fletcher, A. B., Luppino, G. A., Metzger, M. R., \& Moore, C. B. 2001, ApJ, 546, 681

Trager, S. C., King, I. R., \& Djorgovski, S. 1995, AJ, 109, 218

Tremaine, S. D., Ostriker, J. P., \& Spitzer, L. 1975, ApJ, 196, 407

Tully, R. B., \& Pierce, M. J. 2000, ApJ, 533, 744

van der Kruit, P. C., \& Searle, L. 1981, A\&A, 95, 105

Walcher, C.-J., Boeker, T., Charlot, S., Ho, L. C., Rix, H.-W., Rossa, J., Shields, J. C., \& van der Marel, R. P. 2006, ApJ, 649, 692

Walcher, C.-J., et al. 2005, ApJ, 618, 237

Wehner, E., \& Harris, W. 2006, ApJ, 644, L17 